\title{
Quintessential Kination and Thermal Production of Gravitinos AND Axinos
}

\author{
M.E. Gómez ${ }^{\mathrm{a}}$, S. Lola ${ }^{\mathrm{b}}$, C. PAllis ${ }^{\mathrm{a}}$ ANd J. Rodríguez-Quintero ${ }^{\mathrm{a}}$ \\ a Departamento de Fisica Aplicada, \\ Facultad de Ciencias Experimentales, \\ Universidad de Huelva, 21071 Huelva, SPAIN \\ ${ }^{\mathrm{b}}$ Department of Physics, University of Patras, \\ Panepistimioupolis, GR-26500 Patras, GREECE \\ e-mail addresses: mario.gomez@dfa.uhu.es, magda@physics.upatras.gr, \\ kpallis@gen.auth.gr, jose.rodriguez@dfaie.uhu.es
}

\begin{abstract}
The impact of a kination-dominated phase generated by a quintessential exponential model on the thermal abundance of gravitinos and axinos is investigated. We find that their abundances become proportional to the transition temperature from the kination to the radiation era; since this temperature is significantly lower than the initial ("reheating") temperature, the abundances decrease with respect to their values in the standard cosmology. For values of the quintessential energy-density parameter close to its upper bound, on the eve of nucleosynthesis, we find the following: (i) for unstable gravitinos, the gravitino constraint is totally evaded; (ii) If the gravitino is stable, its thermal abundance is not sufficient to account for the cold dark matter of the universe; (iii) the thermal abundance of axinos can satisfy the cold dark matter constraint for values of the initial temperature well above those required in the standard cosmology. A novel calculation of the axino production rate by scatterings at low temperature is also presented.
\end{abstract}

Keywords: Cosmology, Dark Energy, Dark Matter

PACS Codes: 98.80.Cq, 98.80.-k, 95.35.+d

Published in J. Cosmol. Astropart. Phys. 01, 027 (2009) 


\section{Contents}

1 INTRODUCTION 1

2 The Quintessential Exponential Model $\quad 3$

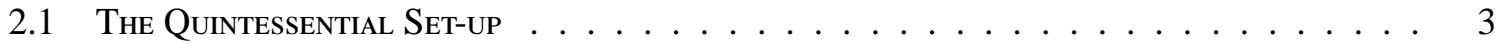

2.2 IMPOSED REQUIREMENTS $\ldots \ldots \ldots \ldots \ldots \ldots \ldots$

2.3 The Quintessential Evolution $\ldots \ldots \ldots \ldots \ldots \ldots \ldots \ldots$

2.4 The Allowed Parameter Space . . . . . . . . . . . . . . . . . . . . . . 7

3 Thermal Abundance of SUSY $e$-WIMPs $\quad 8$

3.1 The BoltzMann Equation $\ldots \ldots \ldots \ldots \ldots \ldots \ldots$

3.2 Numerical Solution $\ldots \ldots \ldots \ldots \ldots \ldots \ldots$

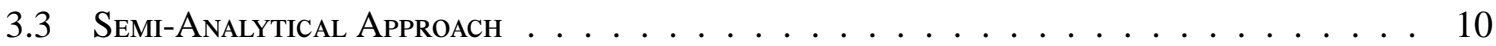

3.4 Numerical Versus Semi-Analytical Results . . . . . . . . . . . . . . . . . . . . 12

4 Kination and Gravitino Thermal Abundance 13

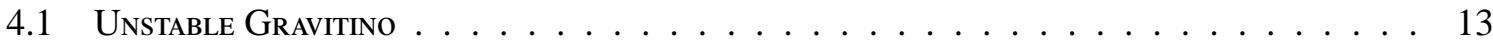

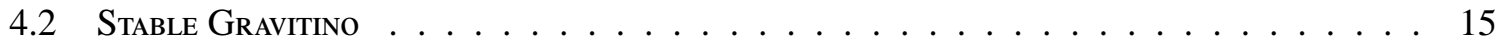

5 Kination and Axino Thermal Abundance 16

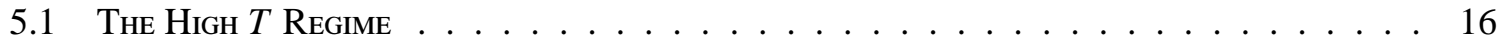

5.2 The Low $T$ Regrme $\ldots \ldots \ldots \ldots \ldots \ldots \ldots \ldots \ldots$

6 Conclusions 19

\begin{tabular}{l|l} 
A Axino Production via Scatterings at Low Temperature & 21
\end{tabular}

\section{INTRODUCTION}

A plethora of recent data [1,2] indicates [3] that the two major components of the present universe are Cold Dark Matter (CDM) and Dark Energy (DE) with density parameters [1]

(a) $\Omega_{\mathrm{CDM}}=0.214 \pm 0.027$ and (b) $\Omega_{\mathrm{DE}}=0.742 \pm 0.03$

at $95 \%$ confidence level (c.1.). Identifying the nature of these two unknown substances, is one of the major challenges in contemporary cosmo-particle theories.

The DE component can be explained by modifying the standard cosmology (SC) via the introduction of a slowly evolving scalar field called quintessence [4] (for reviews, see Ref. [5]). An open possibility in this scenario is the existence of an early kination dominated (KD) era [6], where the universe is dominated by the kinetic energy of the quintessence field; this period is an indispensable ingredient of quintessential inflationary scenaria [7, 8, 9]. During this era, the expansion rate of the universe is larger compared to its value during the usual radiation domination (RD) epoch. This implies that the relic abundance of the weakly interacting massive particles (WIMPs) can be significantly enhanced with respect to (w.r.t) its value in the SC $[10,11,12,13]$, provided that they decouple from the thermal bath during the KD era. Further phenomenological implications of this effect for the future collider or astrophysics experiments have also been studied [14].

WIMPs are the most natural candidates [15] to account for the second major component of the present universe, the CDM. Among them, the most popular is the lightest neutralino $[16,17]$ which 
turns out to be the lightest supersymmetric particle (LSP) in a sizeable fraction of the parameter space of sypersymmetric (SUSY) models and therefore, stable under the assumption of the conservation of $R$-parity. However, SUSY theories predict the existence of even more weakly interacting massive particles, known as $e$-WIMPs [18] which can naturally play the role of LSP. These are the gravitino, $\widetilde{G}$, and the axino, $\tilde{a}$ ( $\widetilde{G}$ is the spin-3/2 fermionic SUSY partner of the graviton, and $\tilde{a}$ the spin- $1 / 2$ fermionic SUSY partner of the axion which arises in SUSY extensions [19] of the Peccei-Quinn (PQ) solution [20] to the strong CP problem). As their name indicates, the interaction rates of gravitinos and axinos are extremely weak, since they are respectively suppressed by the reduced Planck scale, $m_{\mathrm{P}}=M_{\mathrm{P}} / \sqrt{8 \pi}\left(M_{\mathrm{P}}=1.22 \times 10^{19} \mathrm{GeV}\right.$ being the Planck mass $)$ and by the axion decay constant, $f_{a} \sim\left(10^{10}-10^{12}\right) \mathrm{GeV}$ (for a review, see Ref. [21]).

Due to the weakness of their interactions, $e$-WIMPs depart from chemical equilibrium very early ( $\widetilde{G}$ at a energy scale close to $m_{\mathrm{P}}$ and $\tilde{a}$ close to $f_{a}$ ) and we expect that their relic density (created due to this early decoupling) is diluted by the primordial inflation. However, they can be reproduced in the following ways: (i) in the thermal bath, through scatterings [22, 23, 24, 25, 26, 27] and decays $[26,28,29]$ involving superpartners, and (ii) non-thermally [30, 31], from the out-of-equilibrium decay of the next-to-LSP (NLSP). In this paper we do not consider the possible non-thermal production of $e$-WIMPs, since this mechanism is highly model dependent (i.e., it is sensitive to the type and decay products of the NLSP). As a consequence, we do not consider either the out-of-equilibrium decay of the one $e$-WIMP to the other (as in the case of Ref. [32], where $\widetilde{G}$ is the NLSP and $\tilde{a}$ the LSP). In all these cases, extra restrictions have to be imposed in order not to jeopardize the success of the standard Big Bang Nucleosynthensis (NS).

The latter requirement has to be satisfied also for unstable $\widetilde{G}$. This restriction imposes a tight upper bound on the initial ("reheating") temperature, $T_{\mathrm{I}}$, of the universe in the SC $[22,23,33,34,35]$. On the other hand, if one of the $e$-WIMPs is a stable LSP, it has to obey the CDM constraint. In particular, its relic density $\Omega_{X} h^{2}$ has to be confined in the region [1]

$$
\text { (a) } 0.097 \lesssim \Omega_{X} h^{2} \lesssim 0.12 \text { for (b) } 10 \mathrm{keV} \leq m_{X} \leq m_{\mathrm{NLSP}}
$$

where $m_{\mathrm{NLSP}}$ is the mass of the NLSP. Let us note, in passing, that the lower bound of Eq. (1.2a) is valid under the assumption that CDM is entirely composed by $X$ 's and the abundance of non-thermaly produced $X$ 's is negligible. The lower bound on $m_{X}$ arises from the fact that smaller $m_{X}$ cannot explain [36] the observed early reionization [1]. For about $10 \leq m_{X} / \mathrm{keV} \leq 100, X$ 's may constitute warm dark matter (the mass limits above are to be considered only as indicative).

In this paper we reconsider the creation of a KD era in the context of the exponential quintessential model [37, 38], taking into account restrictions arising from NS, the inflationary scale, the acceleration of the universe and the DE density parameter. Although this model does not possess a tracker-type solution $[11,39]$ in the allowed range of its parameters, it can produce a viable present-day cosmology in conjunction with the domination of an early KD era, for a reasonable region of initial conditions $[40,41,42,43]$. We then investigate the impact of KD on the thermal production of $e$-WIMPs, solving the relevant equations both numerically and semianalytically. We find that the abundance of $e$-WIMPs becomes proportional to the transition temperature from $\mathrm{KD}$ to $\mathrm{RD}$ era, $T_{\mathrm{KR}}$, and decreases w.r.t its value in the SC since $T_{\mathrm{KR}}$ can be much lower than $T_{\mathrm{I}}$. In particular, we consider two cases, depending on whether $T_{\mathrm{KR}}$ is higher - high $T$ regime (HTR) - or lower - low $T$ regime (LTR) - than a threshold $T_{\mathrm{C}} \simeq 10 \mathrm{TeV}$, below which the thermal production of $\tilde{a}$ via the decay of the superpartners becomes important; for this low temperature region, a novel formulae for the production of $\tilde{a}$ 's through scatterings is presented. It turns out that, in this part of the parameter space, $\tilde{a}$ becomes an attractive $\mathrm{CDM}$ candidate within the quintessential kination scenario (QKS).

Modifications to the thermal production of $e$-WIMPs have also been investigated in the context of extra dimensional theories $[44,45]$, where the expansion rate of the universe can be also enhanced 
Quintessential Kination and Thermal Production of Gravitinos and Axinos

w.r.t its value in the SC, due to the presence of an extra term including the brane-tension. However, this increase is more drastic than in the QKS. In addition, the production of $\tilde{a}$ via scatterings at low temperature and via the decay [26] of the SUSY particles has not been taken into account [45].

We start our analysis by reviewing the basic features of the exponential quintessential model in Sec. 2. We then present our numerical and semi-analytical calculations of the thermal abundance of $e$-WIMPs in Sec. 3 and study the parameter space allowed by several requirements for $\widetilde{G}(\mathrm{Sec} .4)$ and for $\tilde{a}$ (Sec. 5). Our conclusions are summarized in Sec. 6. Computational issues on the low temperature $\tilde{a}$-production are discussed in Appendix A.

Throughout the text, brackets are used by applying disjunctive correspondence, natural units ( $\hbar=c=k_{\mathrm{B}}=1$ ) are assumed, the subscript or superscript 0 refers to present-day values (except in the coefficient $V_{0}$ ) and $\log [\ln ]$ stands for logarithm with basis $10[e]$. Moreover, we assume that the domain wall number [21] is equal to 1 .

\section{The Quintessential Exponential Model}

In this section we review the system of equations which governs the quintessential cosmological evolution (Sec. 2.1) and the various observational restrictions that we impose (Sec. 2.2). We then describe the salient features of this evolution in Sec. 2.3 and the allowed parameter space in Sec. 2.4.

\subsection{The Quintessential Set-uP}

We assume the existence of a spatially homogeneous scalar field $q$ (not to be confused with the deceleration parameter q in Sec. 2.2), which obeys the Klein-Gordon equation. In particular,

$$
\ddot{q}+3 H \dot{q}+V_{, q}=0, \text { where } V=V_{0} e^{-\lambda q / m_{\mathrm{P}}}
$$

is the adopted potential for the $q$ field, the subscript , $q$ [dot] stands for derivative w.r.t $q$ [the cosmic time, $t]$ and $H$ is the Hubble expansion parameter,

$$
H=\sqrt{\rho_{q}+\rho_{\mathrm{R}}+\rho_{\mathrm{M}}} / \sqrt{3} m_{\mathrm{P}} \text { with } \rho_{q}=\frac{1}{2} \dot{q}^{2}+V,
$$

the energy density of $q$. The energy density of radiation, $\rho_{\mathrm{R}}$, can be evaluated as a function of the temperature, $T$, while the energy density of matter, $\rho_{\mathrm{M}}$, with reference to its present-day value:

$$
\rho_{\mathrm{R}}=\frac{\pi^{2}}{30} g_{\rho *} T^{4} \text { and } \rho_{\mathrm{M}} R^{3}=\rho_{\mathrm{M}}^{0} R_{0}^{3}
$$

( $R$ being the scale factor of the universe). Assuming no entropy production due to domination of $q$ or any other field, the entropy density, $s$, satisfies the following equations:

$$
s R^{3}=s_{\mathrm{p}} R_{\mathrm{p}}^{3} \text { where } s=\frac{2 \pi^{2}}{45} g_{s *} T^{3} .
$$

Here, the subscript "p" represents a specific reference point at which the quantities $s$ and $R$ are evaluated and $g_{\rho *}(T)\left[g_{s *}(T)\right]$ is the energy [entropy] effective number of degrees of freedom at temperature $T$. The numerical values for these quantities are evaluated using the tables included in micrOMEGAs [49]. As it turns out, $g_{\rho *} \simeq g_{s *}$ for $T>T_{\mathrm{NS}}=1 \mathrm{MeV}$, with values $g_{\rho *}=105.74$ for $T \simeq 1 \mathrm{TeV}$ and $g_{\rho *}=228.75$ for $T>1 \mathrm{TeV}$ assuming the particle content of the Minimal SUSY Standard Model (MSSM). Since the abundances under consideration take their present value on the eve of NS $\left(T \simeq T_{\mathrm{NS}}\right.$ ), we do not insist in a distinction between $g_{\rho *}$ and $g_{s *}$ and set $g_{\rho *}=g_{s *}=g_{*}$. On the contrary, for $T<T_{\mathrm{NS}}$ we obtain in general $g_{\rho *}<g_{s *}$. Taking into account the existence of the cosmic background radiation at present, plus three(almost) massless neutrino species, we get [46] $g_{\rho *}^{0}=3.36$ and $g_{s *}^{0}=3.91$. 
The numerical integration of Eq. (2.3) is facilitated by converting the time derivatives to derivatives w.r.t the logarithmic time [40,43], which is defined as a function of the redshift $z$ :

$$
\tau=\ln \left(R / R_{0}\right)=-\ln (1+z) .
$$

Changing the differentiation and introducing the following dimensionless quantities:

$$
\bar{\rho}_{\mathrm{M}[\mathrm{R}]}=\rho_{\mathrm{M}[\mathrm{R}]} / \rho_{\mathrm{c}}^{0}, \bar{V}_{0}=V_{0} / \rho_{\mathrm{c}}^{0} \text { and } \bar{q}=q / \sqrt{3} m_{\mathrm{P}},
$$

Eq. (2.3) turns out to be equivalent to the system of two first-order equations:

$$
\bar{Q}=\bar{H} \bar{q}^{\prime} \text { and } \bar{H} \bar{Q}^{\prime}+3 \bar{H} \bar{Q}+\bar{V}_{, \bar{q}}=0 \text { with } \bar{H}^{2}=\bar{\rho}_{q}+\bar{\rho}_{\mathrm{R}}+\bar{\rho}_{\mathrm{M}} \text {, }
$$

where prime denotes (unless otherwise stated) derivative w.r.t $\tau$ and the following quantities have been defined:

$$
\bar{V}=\bar{V}_{0} e^{-\sqrt{3} \lambda \bar{q}}, \quad \bar{H}=H / H_{0}, \quad \bar{Q}=Q / \sqrt{\rho_{\mathrm{c}}^{0}} \text { and } \bar{\rho}_{q}=\bar{Q}^{2} / 2+\bar{V} .
$$

In our numerical calculation, we use the following values:

$$
\rho_{\mathrm{c}}^{0}=8.099 \times 10^{-47} h^{2} \mathrm{GeV}^{4} \text { and } H_{0}=2.13 \times 10^{-42} h \mathrm{GeV}
$$

with $h=0.72$. In addition, $\bar{\rho}_{\mathrm{M}}^{0}=0.26$ and $T_{0}=2.35 \times 10^{-13} \mathrm{GeV}$ and from Eq. (2.5), we get $\bar{\rho}_{\mathrm{R}}^{0}=8.04 \times 10^{-5}$.

Eq. (2.9) can be resolved numerically by specifying two initial conditions at a logarithmic time, $\tau_{\mathrm{I}}$, which corresponds to a temperature $T_{\mathrm{I}}$ defined as the maximal $T$ after the end of primordial inflation, assuming instantaneous reheating. We take $q\left(\tau_{\mathrm{I}}\right)=0$ throughout our analysis, without any lose of generality [13] and let as free parameter the value the square root of the kinetic-energy density of $q$ at $\tau_{\mathrm{I}}[13]$

$$
\sqrt{\bar{\rho}_{\mathrm{KI}}}=\bar{Q}\left(\tau_{\mathrm{I}}\right) / \sqrt{2} \simeq \sqrt{\bar{\rho}_{q_{\mathrm{I}}}} \simeq \bar{H}_{\mathrm{I}} .
$$

The last equality holds with great accuracy, since we require a complete domination of kination at early times, as we describe below.

\subsection{IMPOSED REQUIREMENTS}

We impose on our quintessential model a number of requirements which can be described as follows:

2.2.1. The Constraint of the Initial Domination of Kination. As we stress in the introduction, we focus our attention on the range of parameters that ensure an absolute or at least a relative initial domination of the $q$-kinetic energy. This requirement can be quantified as follows:

$$
\Omega_{q}^{\mathrm{I}}=\Omega_{q}\left(T_{\mathrm{I}}\right) \gtrsim 0.5 \text { with } \Omega_{q}=\rho_{q} /\left(\rho_{q}+\rho_{\mathrm{R}}+\rho_{\mathrm{M}}\right)
$$

the quintessential energy-density parameter.

2.2.2. Nucleosynthesis Constraint. The presence of $\rho_{q}$ has to preserve the successful predictions of Big Bang NS which starts at about $\tau_{\mathrm{NS}}=-22.5$ corresponding to $T_{\mathrm{NS}}=1 \mathrm{MeV}$ [47]. Taking into account the most up-to-date analysis of Ref. [47], we adopt a rather conservative upper bound on $\Omega_{q}\left(\tau_{\mathrm{NS}}\right)$, less restrictive than the one of Ref. [48]. In particular, we require:

$$
\Omega_{q}^{\mathrm{NS}}=\Omega_{q}\left(\tau_{\mathrm{NS}}\right) \leq 0.21(95 \% \text { c.l. })
$$

where 0.21 corresponds to additional effective neutrinos species $\delta N_{v}<1.6$ [47]. In the left hand side of Eq. (2.14), we do not consider extra (potentially large [52]) contributions from the energy density of gravitational waves generated during a possible former transition from inflation to KD epoch [7]. The reason for this approach is that, inflation could be driven by another field different from $q$ and therefore, any additional constraint from that period would be highly model dependent. Nevertheless, inflation may provide a useful constraint for the parameters of our model, as we discuss below. 
Quintessential Kination and Thermal Production of Gravitinos and Axinos

2.2.3. Inflationary Constraint. Recent data [1] strongly favors the existence of an inflationary phase in the early universe. Assuming that this phase also generates the power spectrum of the curvature scalar $P_{\mathrm{s}}$ and tensor $P_{\mathrm{t}}$ perturbations, an upper bound on the inflationary potential $V_{\mathrm{I}}$ and consequently on $H_{\mathrm{I}}$ can be obtained, following the strategy of Ref. [53]. More specifically, imposing the conservative restriction $r=P_{\mathrm{t}} / P_{\mathrm{S}} \lesssim 1$, and using the observational normalization of $P_{\mathrm{S}}$ [1], we get

$$
H_{\mathrm{I}} \lesssim \frac{\pi}{\sqrt{2}} m_{\mathrm{P}} P_{\mathrm{S} *}^{1 / 2} \Rightarrow H_{\mathrm{I}} \lesssim 2.65 \times 10^{14} \mathrm{GeV} \Rightarrow \bar{H}_{\mathrm{I}} \lesssim 1.72 \times 10^{56}
$$

where $*$ denotes that $P_{\mathrm{s} *}$ is measured at the pivot scale $k_{*}=0.002 / \mathrm{Mpc}$. As we can see in Sec. 2.4 the constraint on $H_{\mathrm{I}}$ is sufficient to restrict the parameter space of our model. Therefore, we are not obliged to impose other constraint on $\tau_{\mathrm{I}}$, or equivalently on $T_{\mathrm{I}}$, as was the case in Ref. [13].

2.2.4. Coincidence Constraint. The present value of $\rho_{q}, \rho_{q}^{0}$, must be compatible with the preferred range of Eq. (1.1b). This can be achieved by adjusting the value of $\bar{V}_{0}$. Since, this value does not affect crucially our results (especially on the $e$-WIMPs abundances), we fix $\bar{\rho}_{q}^{0}=\rho_{q}^{0} / \rho_{\mathrm{c}}^{0}$ to its central experimental value, demanding:

$$
\Omega_{q}^{0}=\bar{\rho}_{q}^{0}=0.74 \text {. }
$$

2.2.5. Acceleration Constraint. A successful quintessential scenario has to account for the present-day acceleration of the universe, i.e. [1],

$$
-1 \leq w_{q}(0) \leq-0.86 \text { (95\% c.l.) with } w_{q}=\left(\dot{q}^{2} / 2-V\right) /\left(\dot{q}^{2} / 2+V\right)
$$

the barotropic index of the $q$-field. In our case, we are not able to avoid [43] the eternal acceleration $\left(w_{q}^{\mathrm{fp}}>-1 / 3\right.$ see below), which is disfavored by string theory.

Let us also comment on our expectations for the redsift $z_{\mathrm{t}}$ of the transition from deceleration to acceleration, and for the age of the universe, $t_{0}$. Due to observational uncertainties in the measurement of these quantities, we do not impose the data on them as absolute constraint. We estimate $z_{t}$ by solving numerically the equation

$$
\mathrm{q}\left(\tau_{\mathrm{t}}\right)=0 \text {, with } z_{\mathrm{t}}=e^{-\tau_{\mathrm{t}}}-1 \text { and } \mathrm{q}=-\ddot{R} R / \dot{R}^{2}=-1-H^{\prime} / H
$$

the deceleration parameter; $t_{0}$ is also estimated numerically, as follows:

$$
t_{0}=\int_{0}^{R_{0}} d R / R=\left(1 / H_{0}\right) \int_{\tau_{\mathrm{I}}}^{0} d \tau / \bar{H}(\tau)
$$

We obtain $0.78 \lesssim z_{\mathrm{t}} \lesssim 0.84$ and $13.6 \gtrsim t_{0} / \mathrm{Gyr} \gtrsim 13.4$ as $w_{q}(0)$ varies in the range of Eq. (2.17). In both cases, as $w_{q}(0)$ approaches -1 the results on $z_{\mathrm{t}}$ and $t_{0}$ approach their values in the context of the standard power-law cosmological model with CDM and a cosmological constant ( $\Lambda \mathrm{CDM})$. The results on $t_{0}$ are in agreement with observational data [1] (according to which $t_{0}=(13.69 \pm 0.26)$ Gyr at $95 \%$ c.1.). On the other hand, our findings on $z_{\mathrm{t}}$ are marginally consistent with the SNe Ia observations [2] according to which $z_{\mathrm{t}}=0.46 \pm 0.26$ at $95 \% \mathrm{c}$.l. However, it is probably premature to say more than that $z_{\mathrm{t}}$ is around unity (see, e.g., Ref. [54]).

\subsection{The Quintessential Evolution}

The quintessence field $q$ undergoes three phases during its cosmological evolution [8, 13]: 


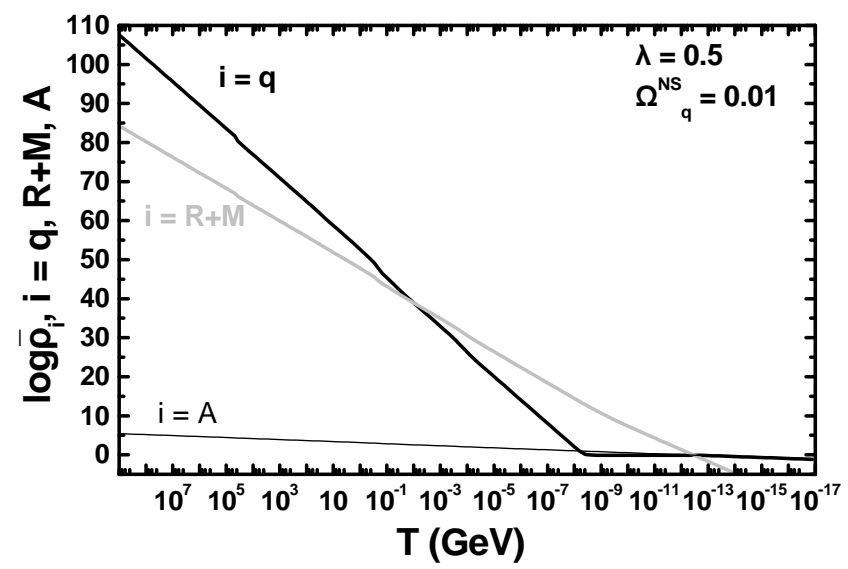

Figure 1: The evolution of the quantities $\log \bar{\rho}_{i}$ with $i=q$ (black lines), $R+M$ (light gray line) and $A$ (thin line) as a function of $T$ for $\lambda=0.5, T_{\mathrm{I}}=10^{9} \mathrm{GeV}$ and $\Omega_{q}^{\mathrm{NS}}=0.01\left(\bar{V}_{0}=2.04 \times 10^{14}\right)$.

- The kinetic-energy dominated phase during which $\rho_{q}$ is essentially given by $\dot{q} / 2 \gg V$; this results to $w_{q} \simeq 1$ and therefore,

$$
\rho_{q}=\rho_{q}^{\mathrm{p}}\left(\frac{R_{\mathrm{p}}}{R}\right)^{6}=\rho_{q}^{\mathrm{p}}\left(\frac{g_{*}}{g_{*}^{\mathrm{p}}}\right)\left(\frac{T}{T_{\mathrm{p}}}\right)^{6}
$$

(where the last equality was extracted using Eq. (2.6)). The subscript or superscript "p" means that the various quantities are evaluated at a reference point $p$ which can be the initial epoch $(p=I)$ or the epoch of the transition between KD and RD era $(p=K R)$ or the eve of NS ( $p=N S)$. Applying Eq. (2.20) for $\mathrm{p}=\mathrm{NS}$, we derive the "transition" temperature $T_{\mathrm{KR}}$ from KD to RD era as follows:

$$
\rho_{q}\left(T_{\mathrm{KR}}\right)=\rho_{\mathrm{R}}\left(T_{\mathrm{KR}}\right) \Rightarrow T_{\mathrm{KR}}=T_{\mathrm{NS}}\left(\frac{g_{*}^{\mathrm{NS}}}{g_{*}^{\mathrm{KR}}}\right)^{1 / 2}\left(\frac{1-\Omega_{q}^{\mathrm{NS}}}{\Omega_{q}^{\mathrm{NS}}}\right)^{1 / 2} .
$$

For $T>T_{\mathrm{KR}}$, the universe undergoes a KD epoch where $H$ is adequately approximated by

$$
H \simeq \frac{1}{\sqrt{3} m_{\mathrm{P}}} \rho_{\mathrm{R}}^{1 / 2}\left(1+\frac{\Omega_{q}^{\mathrm{p}}}{1-\Omega_{q}^{\mathrm{p}}}\left(\frac{g_{*}}{g_{*}^{\mathrm{p}}}\right)\left(\frac{T}{T_{\mathrm{p}}}\right)^{2}\right)^{1 / 2} .
$$

Applying this formula for $\mathrm{p}=\mathrm{NS}$ for any given $T_{\mathrm{I}}$, we get $H_{\mathrm{I}}$ as a function of $\Omega_{q}^{\mathrm{NS}}$. Therefore, we can use $\Omega_{q}^{\mathrm{NS}}$ as a free parameter, instead of $H_{\mathrm{I}}$.

- The frozen-field dominated phase, where the universe becomes RD and $\rho_{q}$ is dominated initially by $\dot{q} / 2$ and subsequently by $V$.

- The attractor dominated phase, where $\rho_{q} \simeq V$ dominates the evolution of the universe, and reaches the late-time attractor energy density:

$$
\left.\bar{\rho}_{\mathrm{A}} \simeq \bar{\rho}_{q}^{0}\left(R / R_{0}\right)^{-3\left(1+w_{q}^{\mathrm{fp}}\right)} \text { with } w_{q}^{\mathrm{fp}}=\lambda^{2} / 3-1 \text { (for } \lambda<\sqrt{3}\right)
$$

the fixed point value of $w_{q}$. Today we obtain a transition from the frozen-field dominated phase to the attractor dominated phase [13,40]. Although this is not a satisfactory solution to the coincidence problem, the observational data can be reproduced for a reasonable set of initial conditions, as we show below.

The cosmological evolution described above is illustrated in Fig. 1 where we plot $\log \bar{\rho}_{i}$ versus $T$ for $T_{\mathrm{I}}=10^{9} \mathrm{GeV}\left(\tau_{\mathrm{I}}=-51.2\right), \Omega_{q}^{\mathrm{NS}}=0.01\left(\bar{H}_{\mathrm{I}}=7.07 \times 10^{53}\right)$ and $\lambda=0.5\left(\bar{V}_{0}=2.04 \times 10^{14}\right)$. For 


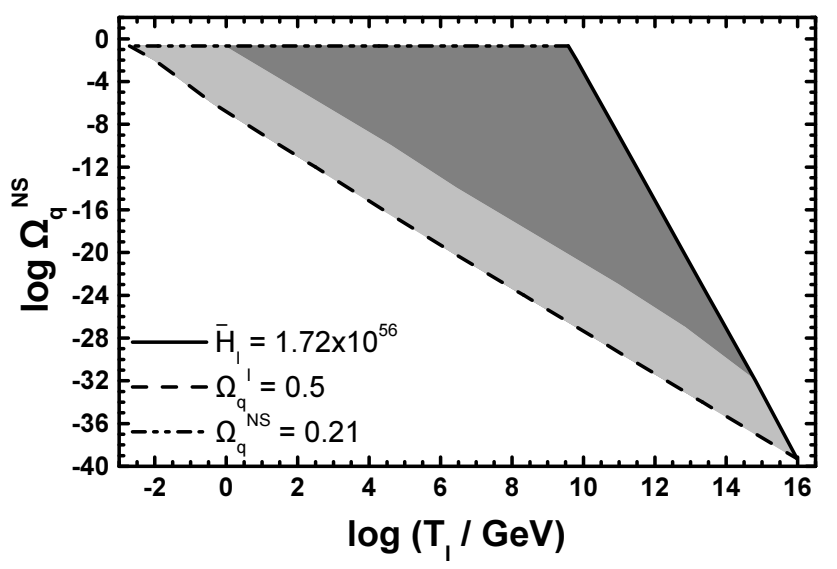

Figure 2: Allowed (gray and lightly grey shaded) region in the $\log T_{\mathrm{I}}-\log \Omega_{q}^{\mathrm{NS}}$ plane by Eqs. (2.13), (2.14) and (2.15). The conventions adopted for the various lines are also shown.

$i=q$ (bold black line), we show $\log \bar{\rho}_{q}$, computed by inserting in Eq. (2.10) the numerical solution of Eq. (2.9). For $i=\mathrm{A}$ (thin black lines), we show $\log \bar{\rho}_{\mathrm{A}}$ derived from Eq. (2.23). For $i=\mathrm{R}+\mathrm{M}$ (light gray line), we show $\log \bar{\rho}_{\mathrm{R}+\mathrm{M}}$, which is the logarithm of the sum of the contributions given by Eq. (2.5). For $T>T_{\mathrm{KR}}=0.0098 \mathrm{GeV}$ we have a KD era. We also obtain $w_{q}(0)=0.957$ whereas $w_{q}^{\mathrm{fp}}=0.916$, $z_{\mathrm{t}}=0.8$ and $t_{0}=13.5 \mathrm{Gyr}$.

\subsection{The Allowed Parameter Space}

The free parameter space of our quintessential model is defined by the following quantities:

$$
\lambda, \tau_{\mathrm{I}} \text { and } \bar{H}_{\mathrm{I}} \text { or equivalently, } \lambda, T_{\mathrm{I}} \text { and } \Omega_{q}^{\mathrm{NS}} \text {. }
$$

Agreement with Eq. (2.17) entails $0<\lambda \lesssim 0.9$ (compare with Ref. [43, 13], where less restrictive upper bounds on $w_{q}(0)$ have been imposed)). This range is independent on $\tau_{\mathrm{I}}$ and $\bar{H}_{\mathrm{I}}\left(\right.$ or $T_{\mathrm{I}}$ and $\Omega_{q}^{\mathrm{NS}}$ ) as is shown in Ref. [13]. Given this fact, we focus on the last two free parameters of our model.

The allowed area in the $\log T_{\mathrm{I}}-\log \Omega_{q}^{\mathrm{NS}}$ plane (which obviously is $\lambda$-independent), is indicated in Fig. 2. In the shaded areas Eqs. (2.13) (2.14) and (2.15) are fulfilled; in particular, in the dark [light] shaded area, $\Omega_{q}^{\mathrm{I}}=1\left[0.5 \lesssim \Omega_{q}^{\mathrm{I}} \lesssim 1\right]$. The left [right] boundary of the allowed regions (indicated by a dashed [solid] line) is derived from Eq. (2.13) [Eq. (2.15)] whereas the upper boundary (indicated by a double dot-dashed line) comes from Eq. (2.14). We observe that the dashed and solid lines are almost straight. This can be understood by deriving the analytic relation between $T_{\mathrm{I}}$ and $\Omega_{q}^{\mathrm{NS}}$ for fixed $H_{\mathrm{I}}$ or $\Omega_{q}^{\mathrm{I}}<1$, namely:

$$
T_{\mathrm{I}}=T_{\mathrm{NS}} \sqrt{\frac{g_{*}^{\mathrm{NS}}}{g_{*}^{\mathrm{I}}} \frac{1-\Omega_{q}^{\mathrm{NS}}}{\Omega_{q}^{\mathrm{NS}}}\left(\frac{\bar{H}_{\mathrm{I}}^{2}}{\bar{\rho}_{\mathrm{R}}^{\mathrm{I}}}-1\right)} \text { and } T_{\mathrm{I}}=T_{\mathrm{NS}} \sqrt{\frac{g_{*}^{\mathrm{NS}}}{g_{*}^{\mathrm{I}}} \frac{1-\Omega_{q}^{\mathrm{NS}}}{\Omega_{q}^{\mathrm{NS}}} \frac{\Omega_{q}^{\mathrm{I}}}{1-\Omega_{q}^{\mathrm{I}}}} .
$$

The first [second] equation can be extracted by calculating $H_{\mathrm{I}}$ through Eq. (2.22) with $\mathrm{p}=\mathrm{NS}$ [p $=\mathrm{I}$ and $\mathrm{p}=\mathrm{NS}$ and eliminating $H_{\mathrm{I}}$ ] and solving the resulting w.r.t $T_{\mathrm{I}}$. Taking the logarithms of the two sides of Eq. (2.24) and given that $\Omega_{q}^{\mathrm{NS}}$ is quite small in the largest part of the available parameter space, we can convince ourselves that the dashed and solid curves in the $\log T_{\mathrm{I}}-\log \Omega_{q}^{\mathrm{NS}}$ plot have to be almost straight. Consequently, for a reasonable set of parameters $\left(\lambda, T_{\mathrm{I}}, \Omega_{q}^{\mathrm{NS}}\right)$, the exponential quintessential model can become consistent with the observational data, in agreement with [13, 40, 43]. 


\section{Thermal Abundance of SUSY $e$-WIMPs}

We assume that any relic abundance of an $e$-WIMP, $X$, due to its decoupling from the thermal bath, is diluted after inflation at an energy scale below $10^{10} \mathrm{GeV}$. We compute its abundance produced through thermal scatterings and decays during the $\mathrm{KD}$ epoch and compare this result with the one obtained in the SC. The relevant equations are presented in Sec. 3.1. In Sec. 3.2 we describe our numerical evaluation and in Sec. 3.3 we derive useful approximate expressions. Their results are compared with the numerical ones in Sec. 3.4.

\subsection{The Boltzmann Equation}

The number density $n_{X}$ of $X$ particles satisfies the Boltzmann equation, which can be collectively written in the following form $[23,26]$ :

$$
\dot{n}_{X}+3 H n_{X}=\frac{1}{2} \sum_{i j}\left\langle(v \sigma)_{i j}\right\rangle n_{i}^{\mathrm{eq}} n_{j}^{\mathrm{eq}}+\sum_{i}\left\langle\Gamma_{i}\right\rangle n_{i}^{\mathrm{eq}} .
$$

Here, $H$ is given by Eq. (2.4) and $n_{i}^{\mathrm{eq}}$ is the equilibrium number density of the particle $i$, which can be adequately calculated in both the relativistic $\left(m_{i} \ll T\right)$ and non-relativistic $\left(m_{i} \gg T\right)$ regime [55]:

$$
n_{i}^{\mathrm{eq}}=\frac{g_{i}}{2 \pi^{2}} m_{i}^{2} T K_{2}\left(m_{i} / T\right)
$$

with $K_{n}$, the modified Bessel function of the second kind of order $n$ and $m_{i}\left[g_{i}\right]$ the mass [the number of degrees of freedom] of the particle $i$.

The quantity $\left\langle(v \sigma)_{i j}\right\rangle$ is the thermal-averaged production rate of $X$ from scatterings in the thermal plasma, the indices $i$ and $j$ run over all particles involved in the initial states of these processes, namely gluons $(g)$, gluinos $(\tilde{g})$, quarks $(q)$ and squarks $(\tilde{q})$, and the factor $1 / 2$ is introduced [23] in order to avoid double counting of the production processes. Formalistically speaking, the term $\left\langle(v \sigma)_{i j}\right\rangle n_{i}^{\mathrm{eq}} n_{j}^{\mathrm{eq}} / 2$ corresponds to the so-called in Refs. $[24,25,27]$ collision term $C_{X} n^{\text {eq2 }}$ and therefore, we can make the replacement:

$$
\frac{1}{2} \sum_{i j}\left\langle(v \sigma)_{i j}\right\rangle n_{i}^{\mathrm{eq}} n_{j}^{\mathrm{eq}}=C_{X} n^{\mathrm{eq} 2} \text { with } C_{X}= \begin{cases}C_{X}^{\mathrm{HT}} & \text { in the HTR, } \\ C_{X}^{\mathrm{LT}} & \text { in the LTR, }\end{cases}
$$

and $n^{\text {eq }}=\zeta(3) T^{3} / \pi^{2}$, the equilibrium number density of the bosonic relativistic species $(\zeta(3) \simeq 1.2$ is the Riemann zeta function of 3 ).

We proceed with the formulas for $C_{X}$ 's in the low (LTR) and high (HTR) $T$ regime. In the relativistic regime $\left(T \gg m_{i}\right), C_{X}$ has been recently recalculated [24, 25, 27] in a consistent gaugeinvariant treatment, using Hard Thermal Loop Approximation (HTLA) technics. The result is

$$
C_{X}^{\mathrm{HT}}= \begin{cases}\frac{3 \pi}{16 \zeta(3) m_{\mathrm{P}}^{2}} \sum_{\alpha=1}^{3}\left(1+\frac{M_{\alpha}^{2}}{3 m_{X}^{2}}\right) c_{\alpha} g_{\alpha}^{2} \ln \left(\frac{k_{\alpha}}{g_{\alpha}}\right) & \text { for } X=\widetilde{G}, \\ \frac{108 \pi g_{a}^{2} g_{3}^{2}}{\zeta(3)} \ln \left(\frac{1.108}{g_{3}}\right) \text { with } g_{a}=\frac{g_{3}^{2}}{32 \pi^{2} f_{a}} & \text { for } X=\tilde{a} .\end{cases}
$$

Here, $g_{\alpha}$ and $M_{\alpha}$ are the gauge coupling constants and gaugino masses respectively, associated with the gauge groups $U(1)_{\mathrm{Y}}, S U(2)_{\mathrm{L}}$ and $S U(3)_{\mathrm{C}},\left(k_{\alpha}\right)=(1.634,1.312,1.271)$ and $\left(c_{\alpha}\right)=(33 / 5,27,72)$. Note that, contrary to the notation of Ref. [25], we use the Grand Unified Theory (GUT) inspired normalization of the hypercharge coupling constant $g_{1}$.

In our study, we calculate $g_{\alpha}$ as a function of the temperature, solving the relevant one loop renormalization group equations with the MSSM particle content. In particular, we have

$$
g_{\alpha}(T)=g_{\mathrm{GUT}}\left(\frac{1}{g_{\mathrm{GUT}}^{2}}-\frac{b_{\alpha}}{8 \pi^{2}} \ln \frac{T}{M_{\mathrm{GUT}}}\right)^{-1 / 2}
$$


Quintessential Kination and Thermal Production of Gravitinos and Axinos

where $\left(b_{\alpha}\right)=(33 / 5,1,-3)$ and $g_{\mathrm{GUT}} \simeq 1 / 24$ is the value of $g$ 's at the GUT scale $M_{\mathrm{GUT}} \simeq 2 \times 10^{16} \mathrm{GeV}$. By adjusting $g_{\mathrm{GUT}}$ and $M_{\mathrm{GUT}}$, we obtain the experimentally acceptable values of $g_{\alpha}$ 's at $M_{Z}$. Below $M_{Z}$, we use the $g_{3}$ running indicated in Ref. [56].

Throughout our analysis we impose universal initial conditions for the gaugino masses i.e., we assume that

$$
M_{\alpha}\left(M_{\mathrm{GUT}}\right)=M_{1 / 2}, \text { with } \alpha=1,2,3 .
$$

The running of the gaugino masses $M_{\alpha}$ can be easily evaluated at one loop by solving the relevant renormalization group equations, which admit an exact solution:

$$
M_{\alpha}(T)=M_{1 / 2}\left(\frac{g_{\alpha}}{g_{\mathrm{GUT}}}\right)^{2}=M_{1 / 2}\left(1-\frac{b_{\alpha}}{8 \pi^{2}} \ln \frac{T}{M_{\mathrm{GUT}}}\right)^{-1}
$$

Under the assumption of Eq. (3.6), the lightest neutralino turns out to be a Bino $(\tilde{B})$, with mass $m_{\tilde{B}} \simeq 0.41 M_{1 / 2}$.

From Eq. (3.4) one easily deduces that $C_{X}^{\mathrm{HT}}$ becomes negative for large enough values of $g_{3}$. Indeed, from Eq. (3.5) we conclude that $g_{3}$ increases as $T$ decreases. In particular, $C_{\tilde{a}}^{\mathrm{HT}}>0$ requires $g_{3} \lesssim 1.108$ or $T>T_{\mathrm{C}}=10^{4} \mathrm{GeV}$. Eq. (3.4), therefore, can be applied self-consistently for $T_{\mathrm{I}}>T_{\mathrm{C}}$ and $T_{\mathrm{KR}}>T_{\mathrm{C}}$. Towards lower values of $T$, the finite masses $m_{i}$, which have been neglected in deriving Eq. (3.4), and $X$-production from decays, start playing an important role. The correct inclusion of these effects is crucial in the case of $\tilde{a}$, since $\Omega_{\tilde{a}} h^{2}$ takes cosmologically interesting values for $T_{\mathrm{I}}<T_{\mathrm{C}}$ and/or $T_{\mathrm{KR}}<T_{\mathrm{C}}$, too. In Appendix A, we present a novel calculation of the term $\left\langle(v \sigma)_{i j}\right\rangle n_{i}^{\mathrm{eq}} n_{j}^{\mathrm{eq}}$ in the case of $\tilde{a}$ and in the non-relativistic regime $\left(T \ll m_{i}\right)$. Our final result can be cast in a form similar to this of Eq. (3.3), with:

$$
C_{\tilde{a}}^{\mathrm{LT}}=\frac{1}{16 T^{5} \zeta(3)^{2}} \sum_{i j} \int_{s_{0}}^{\infty} d s w_{i j}(s) K_{1}\left(\frac{\sqrt{s}}{T}\right) p_{\mathrm{i}}\left(m_{i}, m_{j}\right),
$$

where $s_{0}=\left(m_{i}+m_{j}\right)^{2}$ and the symbols $w_{i j}$ and $p_{\mathrm{i}}$ are defined in Eqs. (A2) and (A3). In particular, $w_{i j}$ is related to the squared amplitudes of the various processes that contribute to $\left\langle(v \sigma)_{i j}\right\rangle$, and are listed in Table 1.

Finally, $\left\langle\Gamma_{i}\right\rangle$ is the thermal-averaged rate of $X$-production from decays in the thermal plasma, which are related to the corresponding decay widths $\Gamma_{i}$ through the formula [55]:

$$
\left\langle\Gamma_{i}\right\rangle=\frac{K_{1}\left(m_{i} / T\right)}{K_{2}\left(m_{i} / T\right)} \Gamma_{i} \Rightarrow\left\langle\Gamma_{i}\right\rangle n_{i}^{\mathrm{eq}}=\frac{g_{i}}{2 \pi^{2}} m_{i}^{2} \Gamma_{i} T K_{1}\left(m_{i} / T\right)
$$

These contributions have been recently calculated in the case of $\widetilde{G}$ in Ref. [29]; however, we do not include them in our calculation since their impact is roughly a factor of two. Such a minor change does not alter our results in any essential way. On the other hand, we do include these contributions in the case of $\tilde{a}$ with $i=\tilde{g}, \tilde{q}$ and $\tilde{B}$, applying the following formulas [28]:

$$
\Gamma_{i}= \begin{cases}4 \frac{g_{a}^{2} g_{3}^{2}}{\pi} m_{\tilde{g}}^{3}\left(1-\frac{m_{\tilde{a}}^{2}}{m_{\tilde{g}}^{2}}\right)^{3} & \text { for } i=\tilde{g}, \\ \frac{3}{2} \frac{g_{a}^{4} g_{3}^{4}}{\pi^{5}} m_{\tilde{q}}\left(m_{\tilde{g}} \ln \frac{f_{a}}{m_{\tilde{g}}}\right)^{2} & \text { for } i=\tilde{q}, \\ \frac{9 g_{1}^{4}}{50 \times 32^{2} \pi^{5} f_{a}^{2}} m_{\tilde{B}}^{3}\left(1-\frac{m_{\tilde{a}}^{2}}{m_{\tilde{B}}^{2}}\right)^{3} & \text { for } \quad i=\tilde{B} .\end{cases}
$$

In the above equations, $g_{3}$ is calculated at a scale equal to $m_{i}$ if $T_{\mathrm{I}}>m_{i}$ or $T_{\mathrm{I}}$ if $T_{\mathrm{I}}<m_{i}$. Obviously, $\Gamma_{\tilde{g}}$ and $\Gamma_{\tilde{q}}$ are much more efficient than $\Gamma_{\tilde{B}}$, due to the presence of $g_{3}$. As we verify numerically, the contribution of these terms to the resulting $\Omega_{X} h^{2}$ becomes important $[28,26,18]$ for $T_{\mathrm{I}}<T_{\mathrm{SUSY}}$ or $T_{\mathrm{KR}}<T_{\mathrm{SUSY}}$, where $T_{\mathrm{SUSY}} \simeq m_{\tilde{q}}$. 


\subsection{Numerical Solution}

In order to find a precise numerical solution to our problem, we have to solve Eq. (3.1) together with Eq. (2.3). To this end, and following the strategy of Sec. 2.1, we introduce the dimensionless quantities:

$$
\bar{n}_{X}=n_{X} /\left(\rho_{\mathrm{c}}^{0}\right)^{3 / 4} \text { and } \bar{n}^{\mathrm{eq}}=n^{\mathrm{eq}} /\left(\rho_{\mathrm{c}}^{0}\right)^{3 / 4} \text {. }
$$

In terms of these quantities (and substituting Eqs. (3.3) and (3.9) into Eq. (3.1)), this takes the following master form, for numerical manipulations:

$$
\bar{H} \bar{n}_{X}^{\prime}+3 \bar{H} \bar{n}_{X}-\bar{C}_{X} \bar{n}^{\mathrm{eq} 2}-\sum_{i} \frac{g_{i}}{2 \pi^{2}} \bar{\Gamma}_{i} \bar{m}_{i}^{2} \bar{T} K_{1}\left(m_{i} / T\right)=0
$$

where $\bar{H}$ is given in Eq. (2.9) and the following quantities have been defined:

$$
\bar{C}_{X}=C_{X} \sqrt{3} m_{\mathrm{P}}\left(\rho_{\mathrm{c}}^{0}\right)^{1 / 4}, \bar{m}_{i}=\frac{m_{i}}{\left(\rho_{\mathrm{c}}^{0}\right)^{1 / 4}}, \bar{T}=\frac{T}{\left(\rho_{\mathrm{c}}^{0}\right)^{1 / 4}} \text { and } \bar{\Gamma}_{i}=\frac{\Gamma_{i}}{H_{0}} .
$$

Eq. (3.12) can be solved numerically with the initial condition $\bar{n}_{X}\left(\tau_{\mathrm{I}}\right) \simeq 0$, where $\tau_{\mathrm{I}}$ corresponds to the initial temperature $T_{\mathrm{I}}$. The integration of Eq. (3.12) runs from $T_{\mathrm{I}}$ down to $T_{\mathrm{NS}}$ (an integration to 0 also gives the same result). We find convenient to single out two cases:

- In the HTR $\left(T_{\mathrm{I}} \gg T_{\mathrm{C}}\right.$ and $T_{\mathrm{KR}} \gg T_{\mathrm{C}}$ ), we integrate Eq. (3.12) with $C_{X}=C_{X}^{\mathrm{HT}}$ from $T_{\mathrm{I}}$ to $T_{\mathrm{NS}}$ (in the integration for $T<T_{\mathrm{C}}$ we take $C_{X}^{\mathrm{HT}}$ frozen at its value at $T \simeq T_{\mathrm{C}}$ ). As it turns out, contributions from Eqs. (3.8) and (3.10) are negligible.

- In the LTR $\left(T_{\mathrm{I}} \ll T_{\mathrm{C}}\right.$ or $T_{\mathrm{KR}} \ll T_{\mathrm{C}}$ ), if (i) $T_{\mathrm{I}}>T_{\mathrm{SUSY}}$, we integrate Eq. (3.12) successively from $T_{\mathrm{I}}$ to $T_{\mathrm{SUSY}}$ with $C_{\tilde{a}}=C_{\tilde{a}}^{\mathrm{HT}}$ and then from $T_{\mathrm{SUSY}}$ to $T_{\mathrm{NS}}$ with $C_{\tilde{a}}=C_{\tilde{a}}^{\mathrm{LT}}$ whereas if (ii) $T_{\mathrm{I}}<T_{\mathrm{SUSY}}$ we integrate Eq. (3.12) from $T_{\mathrm{I}}$ to $T_{\mathrm{NS}}$ with $C_{\tilde{a}}=C_{\tilde{a}}^{\mathrm{LT}}$. It turns out that the contributions from Eq. (3.10) are important only for $T<T_{\mathrm{SUSY}}$. Note that for $T_{\mathrm{SUSY}}<T<T_{\mathrm{C}}$ (where the SUSY particles $i$ with masses $m_{i} \sim T_{\text {SUSY }}$ are relativistic) there is no accurate result for $\left\langle(v \sigma)_{i j}\right\rangle$, since neither HTLA is valid $\left(g_{3}(T)>1.1\right)$ nor our computation in Appendix A is applicable (we consider non-relativistic particles $i$ and $j$ ). However, we believe that the above procedure gives a result which is sufficiently accurate for our proposes, since small variation of $T_{\text {SUSY }}$ (by $10 \%$ ) leaves the result practically unaltered.

The $X$ yield, $Y_{X}=n_{X} / s$, and the relic density of $X, \Omega_{X} h^{2}=\rho_{X}^{0} h^{2} / \rho_{\mathrm{c}}^{0}$ (with $\rho_{X}=m_{X} n_{X}$ ), can be easily found, via the relations [57]:

$$
\text { (a) } Y_{X}^{0}=3.55 \times 10^{-27} \bar{n}_{X}\left(T_{\mathrm{NS}}\right) \text { and (b) } \Omega_{X} h^{2}=2.748 \times 10^{8} Y_{X}^{0} m_{X} / \mathrm{GeV} \text {, }
$$

where $\left(\rho_{\mathrm{c}}^{0}\right)^{3 / 4} / s\left(T_{\mathrm{NS}}\right)=3.55 \times 10^{-27}$ and $s_{0} / \rho_{\mathrm{c}}^{0}=2.748 \times 10^{8} / \mathrm{GeV}$.

\subsection{Semi-Analytical Approach}

The crucial quantity $Y_{X}^{0}$ for the computation of $\Omega_{X} h^{2}$ via Eq. (3.14) can be also derived semianalytically by employing a number of simplifications. We first re-express Eq. (3.1) in terms of the variable $Y_{X}=n_{X} / s$, in order to absorb the dilution term. Indeed [57]

$$
\dot{n}_{X}+3 H n_{X}=\dot{Y}_{X} s=Y_{X}^{\prime} \dot{T} s .
$$

where prime in this section means derivative w.r.t $T$. Employing Eq. (2.6), we obtain:

$$
\dot{s}=-3 H s \Rightarrow \dot{T}=-3 H s / s^{\prime},
$$

where $H$ can be fairly approximated by applying Eq. (2.22) with $\mathrm{p}=\mathrm{KR}$ :

$$
H \simeq \frac{\rho_{\mathrm{R}}^{1 / 2}}{\sqrt{3} m_{\mathrm{P}}}\left(1+r_{q}\right)^{1 / 2}, \text { with } r_{q}=\frac{g_{*}}{g_{*}^{\mathrm{KR}}}\left(\frac{T}{T_{\mathrm{KR}}}\right)^{2}
$$


The presence of $r_{q}>0$ clearly indicates the deviation from the SC, where $r_{q}=0$ and $H=H_{\mathrm{SC}}=$ $\sqrt{\rho_{\mathrm{R}}} / \sqrt{3} m_{\mathrm{P}}$. Inserting Eqs. (3.16) and(3.17) into Eq. (3.15) and substituting in Eq. (3.1), this can be rewritten in terms of the new variables as:

$$
Y_{X}=Y_{\sigma}+Y_{\Gamma}
$$

where $Y_{\sigma}$ and $Y_{\Gamma}$ satisfy the following differential equations:

$$
Y_{\sigma}^{\prime}=-y_{\sigma} / \sqrt{1+r_{q}} \text { and } Y_{\Gamma}^{\prime}=-\sum_{i} y_{\Gamma_{i}} K_{1}\left(m_{i} / T\right) / T^{5} \sqrt{1+r_{q}} .
$$

In the above, we have defined the following quantities:

$$
\begin{aligned}
& y_{\sigma}=y_{X *} Y^{\mathrm{eq} 2} C_{X} \text { and } y_{\Gamma_{i}}=\frac{\pi^{2}}{2 \zeta(3)^{2}} Y^{\mathrm{eq} 2} y_{X *} g_{i} \Gamma_{i} m_{i}^{2} \text { with } Y^{\mathrm{eq}}=\frac{n^{\mathrm{eq}}}{s}, \\
& y_{X *}=\frac{s^{\prime}}{3 H_{\mathrm{SC}}}=\sqrt{\frac{8}{45}} \pi m_{\mathrm{P}} g_{X *}^{1 / 2} \text { and [57] } g_{X *}^{1 / 2}=\frac{g_{s *}}{\sqrt{g_{\rho *}}}\left(1+\frac{T g_{s *}^{\prime}}{3 g_{s *}}\right) .
\end{aligned}
$$

The expression of $H$ as a function of $T_{\mathrm{KR}}$ in Eq. (3.17) enables us to divide the integration of Eqs. (3.19) to two separated domains, since $1 / \sqrt{1+r_{q}}$ can be approximated as follows (compare with Refs. [44, 45]):

$$
1 / \sqrt{1+r_{q}} \simeq \begin{cases}\sqrt{g_{*}^{\mathrm{KR}} / g_{*}}\left(T_{\mathrm{KR}} / T\right) & \text { for } T \gg T_{\mathrm{KR}} \\ 1 & \text { for } T \ll T_{\mathrm{KR}}\end{cases}
$$

Following this simplification, Eq. (3.19) can be resolved trivially. Let us present our results for the HTR and the LTR separately.

3.3.1. THE HTR. In this case $\left(T_{\mathrm{I}} \gg T_{\mathrm{SUSY}}\right.$ and $\left.T_{\mathrm{KR}} \gg T_{\mathrm{SUSY}}\right)$, we find that $Y_{X}^{0} \simeq Y_{\sigma}^{0}$ where

$$
Y_{\sigma}^{0}=\int_{T_{\mathrm{KR}}}^{T_{\mathrm{I}}} d T \sqrt{\frac{g_{*}^{\mathrm{KR}}}{g_{*}}} \frac{T_{\mathrm{KR}}}{T} y_{\sigma}^{\mathrm{HT}}+\int_{0}^{T_{\mathrm{KR}}} d T y_{\sigma}^{\mathrm{HT}}
$$

and $y_{\sigma}^{\mathrm{HT}}=y_{\sigma}\left(C_{X}^{\mathrm{HT}}\right)$. Fixing $C_{X}^{\mathrm{HT}}$ and $g_{*}$ at their initial values in each domain of integration, an analytic formula can be derived, within an $10 \%$ accuracy:

$$
Y_{\sigma}^{0} \simeq \begin{cases}y_{\sigma}^{\mathrm{HT}} T_{\mathrm{I}} & \text { in the SC, } \\ y_{\sigma}^{\mathrm{HT}} \sqrt{g_{*}^{\mathrm{KR}} / g_{*}^{\mathrm{I}}} T_{\mathrm{KR}} \ln \left(T_{\mathrm{I}} / T_{\mathrm{KR}}\right)+y_{\sigma}^{\mathrm{HT}} T_{\mathrm{KR}} & \text { in the QKS. }\end{cases}
$$

From the above expressions, we can easily deduce that $Y_{X}^{0}$ decreases in the QKS w.r.t its value in the $\mathrm{SC}$. This is to be expected, since in the SC it is proportional to $T_{\mathrm{I}}$ whereas, in the QKS, it becomes proportional to $T_{\mathrm{KR}}$ (lower than $T_{\mathrm{I}}$ ). In the latter case, the first term (which is usually ignored in similar estimates $[44,45]$ ) of the right hand side (r.h.s.) of Eq. (3.24), gives an equally important contribution.

3.3.2. THE LTR. In this case $\left(T_{\mathrm{I}} \ll T_{\mathrm{C}}\right.$ or $\left.T_{\mathrm{KR}} \ll T_{\mathrm{C}}\right)$, we find cosmologically interesting solutions only in the case of $\tilde{a}$. We focus on the most intriguing possibility, in which $T_{\mathrm{I}} \gg T_{\mathrm{SUSY}}$ but $T_{\mathrm{KR}} \ll T_{\mathrm{SUSY}}$. In this case, $\Omega_{q}^{\mathrm{NS}}$ takes naturally a value close to its upper bound in Eq. (2.14), as can be verified via Eq. (2.21). We find

$$
\begin{aligned}
Y_{\tilde{a}}^{0} & =Y_{\sigma}^{(1)}+Y_{\sigma}^{(2)}+Y_{\Gamma}^{0} \text { where } \\
Y_{\sigma}^{(1)} & \simeq \int_{T_{\mathrm{SUSY}}}^{T_{\mathrm{I}}} d T \sqrt{\frac{g_{*}^{\mathrm{KR}}}{g_{*}}} \frac{T_{\mathrm{KR}}}{T} y_{\sigma}^{\mathrm{HT}}, \\
Y_{\sigma}^{(2)} & \simeq \int_{T_{\mathrm{KR}}}^{T_{\mathrm{SUSY}}} d T \sqrt{\frac{g_{*}^{\mathrm{KR}}}{g_{*}}} \frac{T_{\mathrm{KR}}}{T} y_{\sigma}^{\mathrm{LT}}+\int_{0}^{T_{\mathrm{KR}}} d T y_{\sigma}^{\mathrm{LT}}, \\
Y_{\Gamma}^{0} & \simeq \sum_{i}\left(\int_{T_{\mathrm{KR}}}^{T_{\mathrm{SUSY}}} d T \sqrt{\frac{g_{*}^{\mathrm{KR}}}{g_{*}}} \frac{T_{\mathrm{KR}}}{T^{6}}+\int_{0}^{T_{\mathrm{KR}}} \frac{d T}{T^{5}}\right) y_{\Gamma_{i}} K_{1}\left(\frac{m_{i}}{T}\right),
\end{aligned}
$$


with $y_{\sigma}^{\mathrm{LT}}=y_{\sigma}\left(C_{X}^{\mathrm{LT}}\right)$. In the SC with $T_{\mathrm{I}}<T_{\mathrm{SUSY}}, Y_{\tilde{a}}^{0}$ can be derived by summing the second terms of the r.h.s. of Eqs. (3.27) and (3.28) and setting $T_{\mathrm{KR}}=T_{\mathrm{I}}$. Unfortunately, finding a general analytical expression for Eqs. (3.26) - (3.28) is not straightforward, mainly because the derivation of $C_{\tilde{a}}^{\mathrm{LT}}$ from Eq. (3.8) requires a double integration of several lengthy squared amplitudes (see Appendix A). However, for the benchmark values of $m_{i}$ used in our analysis:

$$
m_{\tilde{q}}=1 \mathrm{TeV}, m_{\tilde{g}}=1.5 \mathrm{TeV} \text { and } m_{\tilde{B}}=0.3 \mathrm{TeV}
$$

we can write simple empirical relations which reproduce rather accurately our numerical results. We distinguish the following cases:

- In the SC, the main contribution to $Y_{\tilde{a}}^{0}$ arises from the second term of the r.h.s. of Eq. (3.27) [Eq. (3.28)] with $T_{\mathrm{KR}}=T_{\mathrm{I}}$ for $T_{\mathrm{I}}>0.3 \mathrm{TeV}\left[T_{\mathrm{I}}<0.3 \mathrm{TeV}\right]$. Using fitting technics, we get a relation with a $15 \%$ accuracy:

$$
\Omega_{\tilde{a}} h^{2}=A m_{\tilde{a}}\left(1+C T_{\mathrm{I}}\right) e^{-B / T_{\mathrm{I}}} / f_{a}^{2} \text { with } A=1.44 \times 10^{24} \mathrm{GeV},
$$

$B=745.472 \mathrm{GeV}$ and $C=0.001 / \mathrm{GeV}$. The discrepancy can be attributed to the logarithmic factor involved in $\Gamma_{\tilde{q}}-$ see Eq. (3.10). This factor disturbs the dependence of $\Omega_{\tilde{a}} h^{2}$ on $f_{a}$ as written in Eq. (3.30). The origin of the exponential factor is the non-relativistic expansion [55] of $K_{1}(\sqrt{s} / T)\left[K_{1}\left(m_{i} / T\right)\right]$ involved in the definition of $\left\langle(v \sigma)_{i j}\right\rangle\left[\left\langle\Gamma_{i}\right\rangle\right]-$ see Eq. (3.8) [Eq. (3.9)].

- In the QKS, the main contribution to $Y_{\tilde{a}}^{0}$ comes from the first term of the r.h.s. of Eq. (3.27) and therefore, we expect that $\Omega_{\tilde{a}} h^{2}$ is independent of $T_{\mathrm{I}}$. Our results can be reproduced from the following relation which holds with an excellent accuracy:

$$
\Omega_{\tilde{a}} h^{2}=\frac{D}{f_{a}^{2}} \frac{m_{\tilde{a}}}{\sqrt{\Omega_{q}^{\mathrm{NS}}}}=\frac{D}{f_{a}^{2}} \frac{T_{\mathrm{KR}}}{T_{\mathrm{NS}}} \sqrt{\frac{g_{*}^{\mathrm{KR}}}{g_{*}^{\mathrm{NS}}}} m_{\tilde{a}} \text { with } D=9.26 \times 10^{17} \mathrm{GeV}
$$

where we used Eq. (2.21) in the last equality. We observe that $\Omega_{\tilde{a}} h^{2} \propto 1 / \sqrt{\Omega_{q}^{\mathrm{NS}}}$ or $\Omega_{\tilde{a}} h^{2} \propto T_{\mathrm{KR}}$.

We conclude, therefore, that in both the QKS and the SC our calculation of $C_{\tilde{a}}^{\mathrm{LT}}$ is crucial in order to achieve a reliable result for $\Omega_{\tilde{a}} h^{2}$.

\subsection{Numerical Versus Semi-Analytical Results}

The validity of our semi-analytical approach can be tested by comparing its results for $\Omega_{X} h^{2}$ with those obtained by the numerical solution of Eq. (3.12). In addition, useful conclusions can be inferred for the behavior of $\Omega_{X} h^{2}$ as a function of $m_{X}$ and $\Omega_{q}^{\mathrm{NS}}$. Our results are presented in Fig. 3. The lines are drawn applying our numerical code, whereas crosses are obtained by employing the formulas of Sec. 3.3. In Figs. 3-(a) [3-(b)], we display $\Omega_{\widetilde{G}} h^{2}\left[\Omega_{\tilde{a}} h^{2}\right]$ versus $m_{\widetilde{G}}\left[m_{\tilde{a}}\right]$ for $M_{1 / 2}=0.7 \mathrm{TeV}\left[f_{a}=10^{11} \mathrm{GeV}\right]$ and various $\Omega_{q}^{\mathrm{NS}}$ 's indicated on the curves. We take $T_{\mathrm{I}}=10^{9} \mathrm{GeV}$, a value frequently met in the well-motivated models of SUSY hybrid inflation [58].

As we can verify via Eq. (2.21), the results for the HTR are applicable for any $\Omega_{q}^{\text {NS }}$ in Fig. 3-(a) and for $\Omega_{q}^{\mathrm{NS}}=10^{-15}$ in Fig. 3-(b), whereas for the residual $\Omega_{q}^{\mathrm{NS}}$ in Fig. 3-(b) the results for LTH hold; in particular, the crosses are obtained from Eq. (3.23) [Eq. (3.25)] in the HTR [LTR]. In both regimes, $\Omega_{X} h^{2}$ decreases as $\Omega_{q}^{\mathrm{NS}}$ increases, as we expect by combining Eqs. (3.23) and (2.21) for the HTR, and from Eq. (3.31) for the LTR. On the other hand, $\Omega_{\widetilde{G}} h^{2} \propto 1 / m_{\widetilde{G}}$, while $\Omega_{\tilde{a}} h^{2} \propto m_{\tilde{a}}$. This can be understood from the fact that $\Omega_{X} h^{2} \propto m_{X} Y_{X}^{0}$ and $Y_{X}^{0} \propto C_{X}$ or $Y_{X}^{0} \propto \Gamma_{i}$. However $C_{\widetilde{G}}^{\mathrm{HT}} \propto 1 / m_{\widetilde{G}}^{2}$ (for $M_{\alpha}>m_{\widetilde{G}}$ ) whereas $C_{\tilde{a}}^{\mathrm{HT}}, C_{\tilde{a}}^{\mathrm{LT}}$ and $\Gamma_{i}$ are essentially independent on $m_{\tilde{a}}$ (see also Ref. [26]).

In most cases, we observe that the semi-analytical findings approach rather successfully the numerical ones. Let us clarify, however, that the results presented in the following sections, are derived exclusively by our numerical program. 


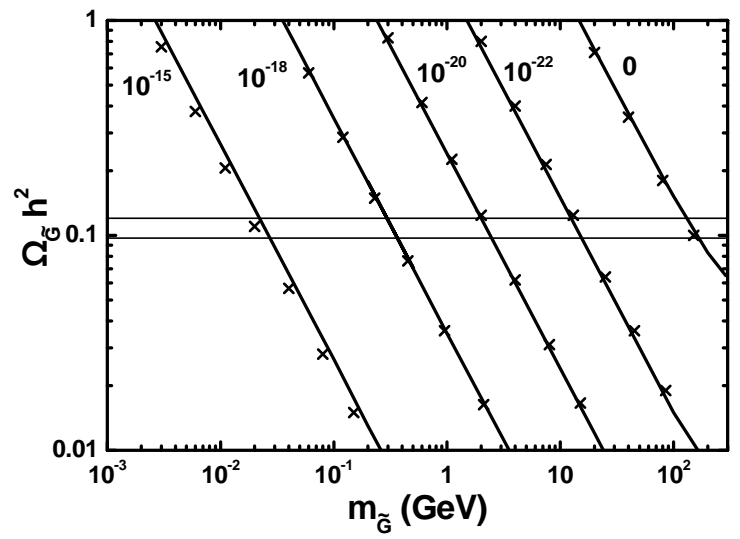

(a)

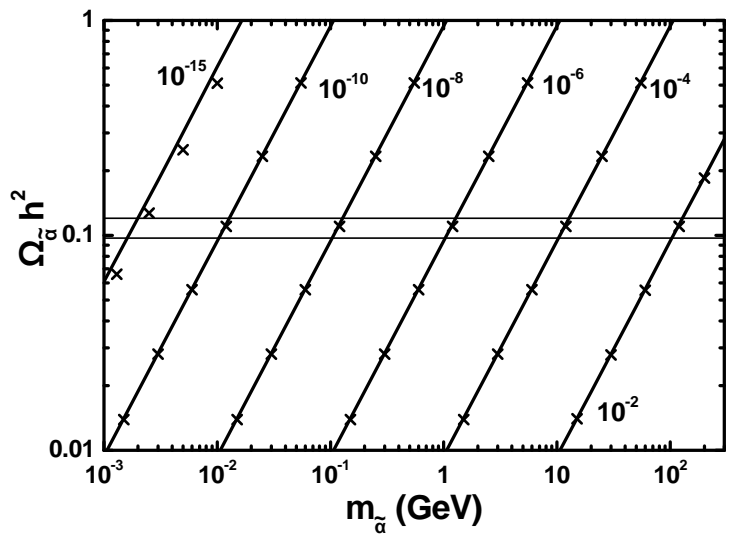

(b)

Figure 3: $\Omega_{X} h^{2}$ as a function of $m_{X}(X=\widetilde{G}[X=\tilde{a}])$ for various $\Omega_{q}^{\mathrm{NS}}$, $s$, indicated on the curves, $T_{\mathrm{I}}=10^{9} \mathrm{GeV}$ and $M_{1 / 2}=0.7 \mathrm{TeV}\left[f_{a}=10^{11} \mathrm{GeV}\right](\mathrm{a}[\mathrm{b}])$. For $\Omega_{q}^{\mathrm{NS}}>10^{-15}$, we take in our computation the values of $m_{i}$ indicated in Eq. (3.29). The solid lines [crosses] are obtained by our numerical code [semi-analytical expressions]. The CDM bounds of Eq. (1.2) are also, depicted by the two thin lines.

\section{Kination and Gravitino Thermal Abundance}

We first examine the impact of a KD era on the thermal abundance of $\widetilde{G}$ and study its cosmological consequences. We discriminate two cases, depending on whether $\widetilde{G}$ is unstable (Sec. 4.1) or stable (Sec. 4.2).

\subsection{Unstable Gravitino}

If $\widetilde{G}$ is unstable, it can decay after the onset of NS, affecting the primordial abundances of the light elements in an unacceptable way. In order to avoid spoiling the success of Big Bang NS, an upper bound on $Y_{\widetilde{G}}$ is to be extracted as a function of $m_{\widetilde{G}}$ and the hadronic branching ratio of $\widetilde{G}, B_{\mathrm{h}}$ $[33,34,35]$. Let us specify some representative values of this constraint, taking into account the most up-to-date analysis of Ref. [33]. In particular, if $\widetilde{G}$ decays mainly to photon and photino, from Fig. 1 of Ref. [33] we can deduce:

$$
Y_{\widetilde{G}}\left(T_{\mathrm{NS}}\right) \lesssim\left\{\begin{array} { l } 
{ 1 0 ^ { - 1 5 } } \\
{ 1 0 ^ { - 1 4 } } \\
{ 1 0 ^ { - 1 3 } }
\end{array} \text { for } m _ { \widetilde { G } } \simeq \left\{\begin{array}{c}
360 \mathrm{GeV} \\
600 \mathrm{GeV} \\
11 \mathrm{TeV}
\end{array} \text { and } B_{\mathrm{h}}=0.001,\right.\right.
$$

whereas if $\widetilde{G}$ decays mainly to gluons and gluinos, from Fig. 2 of Ref. [33] we can deduce:

$$
Y_{\widetilde{G}}\left(T_{\mathrm{NS}}\right) \lesssim\left\{\begin{array} { c } 
{ 1 0 ^ { - 1 5 } } \\
{ 1 0 ^ { - 1 6 } } \\
{ 9 . 6 \times 1 0 ^ { - 1 5 } }
\end{array} \text { for } m _ { \widetilde { G } } \simeq \left\{\begin{array}{l}
200 \mathrm{GeV} \\
680 \mathrm{GeV} \\
10 \mathrm{TeV}
\end{array} \text { and } B_{\mathrm{h}}=1 .\right.\right.
$$

We observe that for $B_{\mathrm{h}}=1$, the upper bound on $Y_{\widetilde{G}}\left(T_{\mathrm{NS}}\right)$ does not exclusively increase with an increase of $m_{\widetilde{G}}$, as in the case for $B_{\mathrm{h}}=0.001$.

In the SC (where no late-time entropy production is expected), setting $M_{1 / 2}=500 \mathrm{GeV}$, we obtain a stringent upper bound on $T_{\mathrm{I}}$ :

$$
\begin{aligned}
& T_{\mathrm{I}} \lesssim\left\{\begin{array} { l } 
{ 2 . 3 \times 1 0 ^ { 6 } \mathrm { GeV } } \\
{ 4 \times 1 0 ^ { 7 } \mathrm { GeV } } \\
{ 6 \times 1 0 ^ { 8 } \mathrm { GeV } }
\end{array} \text { for } m _ { \widetilde { G } } \simeq \left\{\begin{array}{c}
360 \mathrm{GeV} \\
600 \mathrm{GeV} \\
11 \mathrm{TeV}
\end{array} \text { and } B_{\mathrm{h}}=0.001\right.\right. \text {, or } \\
& T_{\mathrm{I}} \lesssim\left\{\begin{array}{l}
8.5 \times 10^{5} \mathrm{GeV} \\
3.1 \times 10^{5} \mathrm{GeV} \text { for } m_{\widetilde{G}} \simeq\left\{\begin{array}{c}
200 \mathrm{GeV} \\
5.4 \times 10^{7} \mathrm{GeV}
\end{array}\right. \text { 傮 } \\
10 \mathrm{TeV}
\end{array} \text { and } B_{\mathrm{h}}=1 .\right.
\end{aligned}
$$




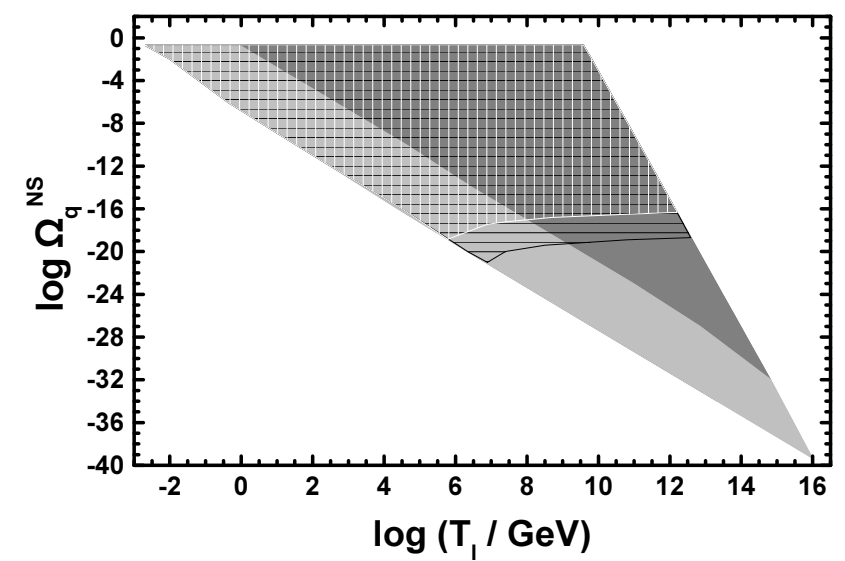

Figure 4: Areas in the $\log T_{\mathrm{I}}-\log \Omega_{q}^{\mathrm{NS}}$ plane that are simultaneously allowed by the quintessential requirements (gray and lightly gray shaded area) - Eqs. (2.13), (2.14) and (2.15) - and the gravitino constraint for $m_{\widetilde{G}}=M_{1 / 2}=500 \mathrm{GeV}$ and $B_{\mathrm{h}}=0.001$ (black lined area) or $B_{\mathrm{h}}=1$ (white lined area).

Clearly the upper bound on $T_{\mathrm{I}}$ becomes significantly more restrictive for large $B_{\mathrm{h}}$ 's and low $m_{\widetilde{G}}$ 's.

In the QKS, if we set $T_{\mathrm{I}}=10^{9} \mathrm{GeV}$ and $M_{1 / 2}=500 \mathrm{GeV}$ we can obtain a lower bound on $\Omega_{q}^{\mathrm{NS}}$ which can be transformed to an upper bound on $T_{\mathrm{KR}}$ via Eq. (2.21). In particular,

- For $B_{\mathrm{h}}=0.001$,

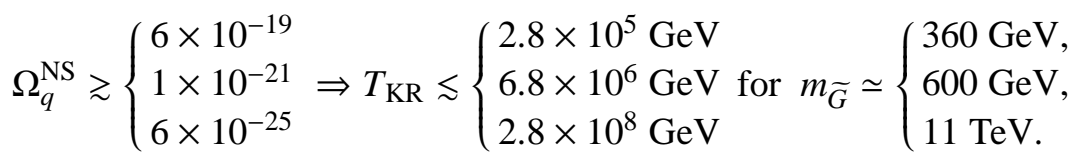

- For $B_{\mathrm{h}}=1$,

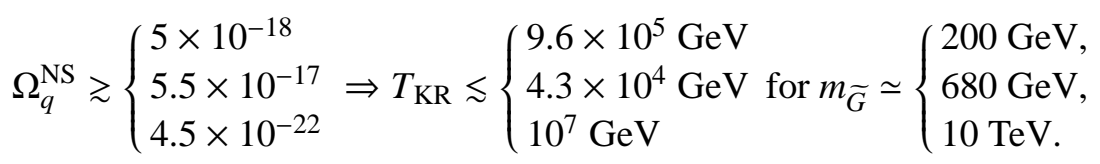

The importance of a KD era in avoiding the gravitino constraint can also be induced by Fig. 4, where, in contrast to our previous approach, $T_{\mathrm{I}}$ is now variable, whereas $m_{\widetilde{G}}$ is fixed to a representative value. In Fig. 4, we show the regions in the $\log T_{\mathrm{I}}-\log \Omega_{q}^{\mathrm{NS}}$ plane that are allowed by the quintessential requirements (see Fig. 2), for $m_{\widetilde{G}}=M_{1 / 2}=500 \mathrm{GeV}$ and $B_{\mathrm{h}}=0.001$ (black lined area) or $B_{\mathrm{h}}=1$ (white lined area). We observe that for $B_{\mathrm{h}}=0.001$ the required minimal $\Omega_{q}^{\mathrm{NS}}$ is lower that in the case of $B_{\mathrm{h}}=1$. This is because for $B_{\mathrm{h}}=0.001$ we impose $Y_{\widetilde{G}}\left(T_{\mathrm{NS}}\right) \lesssim 2 \times 10^{-15}$, whereas for $B_{\mathrm{h}}=1$, we impose $Y_{\widetilde{G}}\left(T_{\mathrm{NS}}\right) \lesssim 2 \times 10^{-16}$ (in accordance with Figs 1 and 2 of Ref. [33]). As a consequence, the maximal allowed $T_{\mathrm{KR}} \simeq\left(4.8 \times 10^{5}-6.8 \times 10^{6}\right) \mathrm{GeV}$ for $B_{\mathrm{h}}=0.001$ is higher than the one $\left(4.6 \times 10^{4}-5.7 \times 10^{5}\right) \mathrm{GeV}$ allowed for $B_{\mathrm{h}}=1$. Finally, we observe that the minimal $\Omega_{q}^{\mathrm{NS}}$ depends very weakly on $T_{\mathrm{I}}$.

We can conclude, therefore, that the gravitino constraint can be totally eluded in the QKS, even for tiny values of $\Omega_{q}^{\mathrm{NS}}$. 


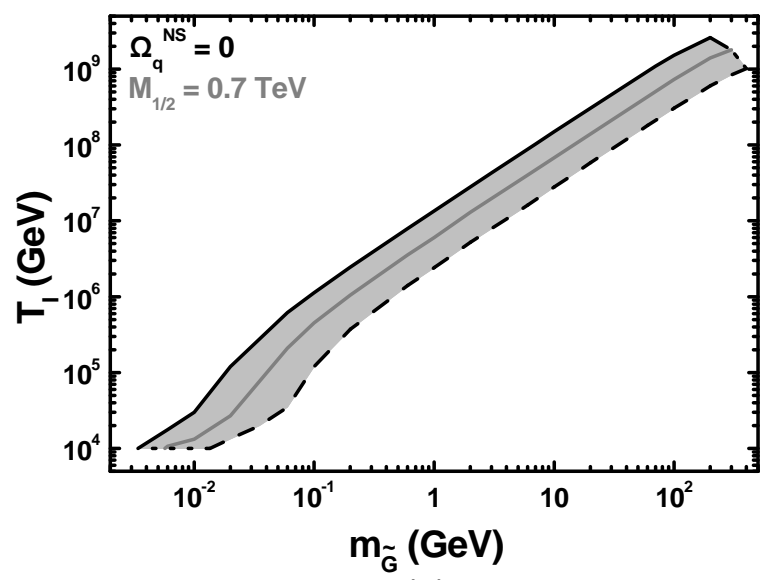

(a)

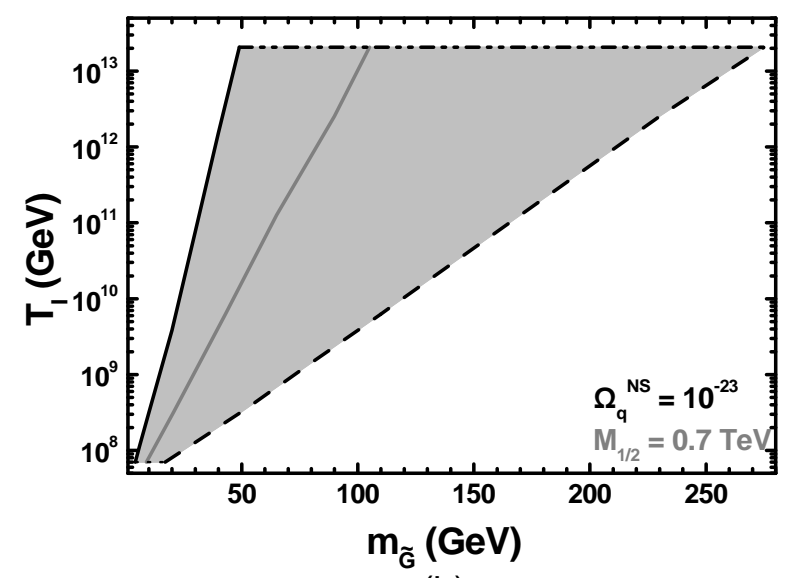

(b)

$$
\begin{aligned}
& \Omega_{\tilde{G}} h^{2}=0.12 \quad \cdots-m_{\tilde{G}}=m_{\tilde{B}} \\
& -\Omega_{\hat{G}^{2}} h^{2}=0.11 \quad \cdots-\bar{H}_{1}=1.7 \times 10^{56} \\
& \begin{array}{lll}
- & \Omega_{\widehat{G}} h^{2}=0.097 & \cdots \cdot g_{3} \sim 1
\end{array} \\
& \cdots \cdots \Omega_{\mathrm{q}}=0.5
\end{aligned}
$$

Figure 5: Allowed (lightly gray shaded) regions in the $m_{\widetilde{G}}-T_{\text {I }}$ plane for $\widetilde{G}-C D M$ with $0.5 \leq M_{1 / 2} / \mathrm{TeV} \leq$ 1 in the (a) $S C\left(\Omega_{q}^{\mathrm{NS}}=0\right)$ and (b) $Q K S$ with $\Omega_{q}^{\mathrm{NS}}=10^{-23}$. The conventions adopted for the various lines are also shown.

\subsection{Stable Gravitino}

$\widetilde{G}$ can be stable if it is the LSP within SUSY models with R-parity conservation. In this case, it constitutes a good CDM candidate, provided $\Omega_{\widetilde{G}} h^{2}$ satisfies Eq. (1.2). Imposing this condition constrains the free parameters, which in this case are $m_{\widetilde{G}}, T_{\mathrm{I}}, M_{1 / 2}$ and $\Omega_{q}^{\mathrm{NS}}$.

In Fig. 5 we present the allowed regions (lightly gray shaded) in the $m_{\widetilde{G}}-T_{I}$ plane in the (a) SC $\left(\Omega_{q}^{\mathrm{NS}}=0\right)$ and (b) QKS with $\Omega_{q}^{\mathrm{NS}}=10^{-23}$, letting $M_{1 / 2}$ vary in the interval $(0.5-1) \mathrm{TeV}$. The black solid [dashed] lines correspond to the upper [lower] bound on $\Omega_{\widetilde{G}} h^{2}$ in Eq. (1.2a), whereas the gray solid lines have been obtained by fixing $\Omega_{\widetilde{G}} h^{2}$ to its central value in Eq. (1.2a) for $M_{1 / 2}=0.7 \mathrm{TeV}$. In practice, the solid [dashed] line is constructed for $M_{1 / 2}=0.5 \mathrm{TeV}\left[M_{1 / 2}=1 \mathrm{TeV}\right]$. This is because $\Omega_{\widetilde{G}} h^{2} \propto T_{\mathrm{I}} M_{\alpha}^{2} / m_{\widetilde{G}}\left[\Omega_{\widetilde{G}} h^{2} \propto \ln T_{\mathrm{I}} M_{\alpha}^{2} / m_{\widetilde{G}}\right]$ in the SC [QKS] as deduced by Eqs. (3.24) and (3.4).

The upper boundary curve (dot-dashed line) in Fig. 5-(a) results from the requirement that $\widetilde{G}$ is the LSP and thus is lighter than $\tilde{B}$. Note that $m_{\tilde{B}}$ varies from 205 to $411 \mathrm{GeV}$ for $M_{1 / 2}$ in the interval $(0.5-1) \mathrm{TeV}$. On the other hand, the upper boundary curve (double dot-dashed line) in Fig. 5-(b) comes from the upper bound on $\bar{H}_{\mathrm{I}}$ in Eq. (2.15). The lower boundary curve (dotted [thin] line) in Fig. 5-(a) [Fig. 5-(b)] arises from the saturation of $g_{3}<1$ [Eq. (2.13)]. Note that towards lower $T$ 's the preferred $m_{\widetilde{G}}$ tends to almost unnaturally small values. We conclude therefore, that the LTR is not cosmologically interesting in the case of $\widetilde{G}$-CDM.

From Fig. 5-(a) we observe that in the SC $\left(\Omega_{q}^{\mathrm{NS}}=0\right)$ somehow larger $T_{\mathrm{I}}$ 's than in the case with unstable $\widetilde{G}$ are allowed (compare, e.g., with Eq. (4.3)). Much larger $T_{\mathrm{I}}$ 's and $m_{\widetilde{G}}$ 's are allowed in the QKS - see Fig. 5-(b). However, in this case, we are obliged to fine tune $\Omega_{q}^{\mathrm{NS}}$ to a very low value $10^{-23}$ $\left(T_{\mathrm{KR}}=7 \times 10^{7} \mathrm{GeV}\right)$, in order to obtain acceptable $\Omega_{\widetilde{G}} h^{2}$. We consider such a low $\Omega_{q}^{\mathrm{NS}}$ as unnatural and thus, conclude that $\widetilde{G}$ is not a good CDM candidate within the QKS. 


\section{Kination and Axino Thermal Abundance}

We now turn to $\tilde{a}$, as the main candidate for CDM in the universe. The production of $\tilde{a}$ is usually accompanied by the production of a scalar SUSY partner, the s-axion; this may undergo out-ofequilibrium decays [59], producing entropy and therefore, diluting any prexisting $\Omega_{\tilde{a}} h^{2}$. In our analysis, we assume that this is not the case [26]. Moreover, we limit ourselves to $T_{\mathrm{I}} \leq f_{a}$ since for larger $T_{\mathrm{I}}$ 's the PQ symmetry [20] is restored and so, no particle from the axion supermultiplet has been produced.

Let us initially derive the temperature $T_{\mathrm{D}}$ at which $\tilde{a}$ decouples from the thermal bath in the SC and QKS. The $\tilde{a}$-decoupling occurs when

$$
H\left(T_{\mathrm{D}}\right) \simeq \Gamma_{\tilde{a}}\left(T_{\mathrm{D}}\right), \text { where } \Gamma_{\tilde{a}}=\left\langle(v \sigma)_{\tilde{a}}\right\rangle n^{\mathrm{eq}} \sim 6 N_{\mathrm{F}}\left(N_{3}^{2}-1\right) g_{a}^{2} g_{3}^{2} n^{\mathrm{eq}} / 2
$$

is an update [60] of the interaction rate which maintains $\tilde{a}$ 's in chemical equilibrium with the thermal bath (we take $N_{\mathrm{F}}=12$ and $N_{3}=3$ as explained in Appendix A). Similarly to the previous discussion, we distinguish two cases:

- In the SC, solving Eq. (5.1) w.r.t $T_{\mathrm{D}}$ (after replacing $H$ by its expression in Eq. (3.17) for $r_{q}=0$ ), we find

$$
\begin{aligned}
& T_{\mathrm{D}}^{\mathrm{SC}} \simeq \frac{\pi^{4} g_{*}^{\mathrm{D}}}{8640 \sqrt{3} m_{\mathrm{P}} g_{a}^{2} g_{3}^{2} \zeta(3)} \simeq 10^{8} \mathrm{GeV} \text { for } f_{a}=10^{11} \mathrm{GeV} \text { and } \\
& 6 \times 10^{5} \lesssim T_{\mathrm{D}}^{\mathrm{SC}} / \mathrm{GeV} \lesssim 2 \times 10^{10} \text { for } 10^{10} \leq f_{a} / \mathrm{GeV} \leq 10^{12}
\end{aligned}
$$

- In the QKS (after replacing $H$ in Eq. (5.1) by its expression in Eq. (3.17) with $r_{q}$ given by Eq. (3.22)), we find

$$
\begin{aligned}
& H>\Gamma_{\tilde{a}} \text { for } T_{\mathrm{KR}}<T_{\mathrm{D}}^{\mathrm{SC}} \text { or } \Omega_{q}^{\mathrm{NS}} \gtrsim \frac{g_{*}^{\mathrm{NS}}}{g_{*}^{\mathrm{D}}}\left(\frac{T_{\mathrm{NS}}}{T_{\mathrm{D}}^{\mathrm{SC}}}\right)^{2} \\
& \Rightarrow \Omega_{q}^{\mathrm{NS}} \gtrsim\left\{\begin{array}{cl}
1 \times 10^{-19} & \text { for } f_{a}=10^{10} \mathrm{GeV}, \\
3.5 \times 10^{-24} & \text { for } f_{a}=10^{11} \mathrm{GeV}, \\
1 \times 10^{-28} & \text { for } f_{a}=10^{12} \mathrm{GeV} .
\end{array}\right.
\end{aligned}
$$

These values were extracted numerically without the approximation of Eq. (3.22) and therefore, are less restrictive that the result of the analytical estimate in Eq. (5.4). Note that the constraints on $\Omega_{q}^{\mathrm{NS}}$ are independent on $T_{\mathrm{I}}$.

As we already emphasize in Sec. 3 we expect that the primordial (i.e., due to the $\tilde{a}$-decoupling) $\tilde{a}$ yield [27] $\left(Y_{\tilde{a}}^{\mathrm{D}}=\left(3 n^{\mathrm{eq}} / 2 s\right)\left(T_{\mathrm{D}}\right) \simeq 1.8 \times 10^{-3}\right)$ is diluted by the entropy release during reheating of the universe to a temperature $T_{\mathrm{I}}$. Under this assumption, we have to ensure that $T_{\mathrm{I}}<T_{\mathrm{D}}^{\mathrm{SC}}\left[T_{\mathrm{KR}}<T_{\mathrm{D}}^{\mathrm{SC}}\right]$ constructing the regions where $\tilde{a}$ is a viable CDM-candidate in the SC [QKS].

Our results for the HTR and the LTR are analyzed separately in the following. Let us remind that the discrimination between the two regimes (HTR or LTR) depends on the hierarchy not only between $T_{\mathrm{I}}$ and $T_{\mathrm{C}}$ but also between $T_{\mathrm{KR}}$ and $T_{\mathrm{C}}$. In the $\mathrm{SC}$ we have $T_{\mathrm{I}} \gg T_{\mathrm{C}}\left[T_{\mathrm{I}} \ll T_{\mathrm{C}}\right.$ ] for the HTR [LTR] (see Sec. 5.1 [Sec. 5.1]). In the QKS we take $T_{\mathrm{I}} \gg T_{\mathrm{C}}$ and $T_{\mathrm{KR}}>T_{\mathrm{C}}$ for the HTR (see Sec. 5.1), but we consider as more natural choice (motivated by the majority [58] of the inflationary models) to take $T_{\mathrm{I}} \gg T_{\mathrm{C}}$ and $T_{\mathrm{KR}}<T_{\mathrm{C}}$ for LTR (see Sec. 5.2). The dependence of our results on the variation of $T_{\mathrm{I}}$ in the QKS is also studied in Sec. 5.2.

\subsection{The High $T$ Regime}

In this case the HTLA works well and therefore the comparison with the case of $\widetilde{G}$-CDM is straightforward; the free parameters in the present case are: $m_{\tilde{a}}, f_{a}, T_{\mathrm{I}}$ and $\Omega_{q}^{\mathrm{NS}}$. 


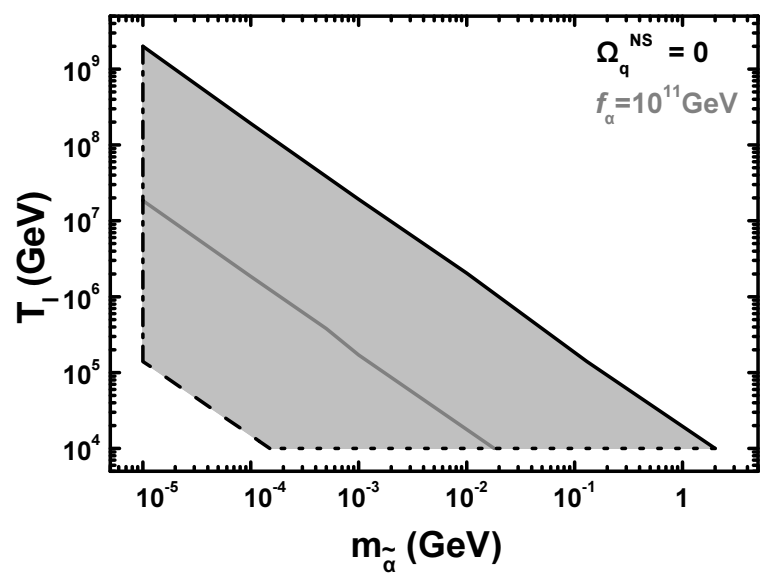

(a)

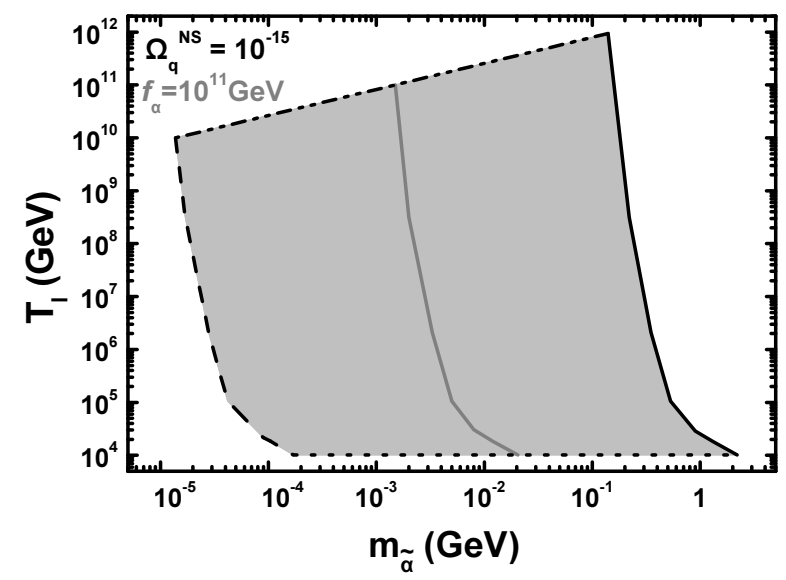

(b)

Figure 6: Allowed (lightly gray shaded) regions for $\tilde{a}-C D M$ in the HTR and $m_{\tilde{a}}-T_{\mathrm{I}}$ plane with $10^{10} \leq f_{a} / \mathrm{GeV} \leq 10^{12}$ for the (a) $S C\left(\Omega_{q}^{\mathrm{NS}}=0\right)$ and (b) $Q K S$ with $\Omega_{q}^{\mathrm{NS}}=10^{-15}$. The conventions adopted for the various lines are also shown.

In Fig. 6 we display the allowed regions (lightly gray shaded) in the $m_{\tilde{a}}-T_{\mathrm{I}}$ plane in the (a) $\mathrm{SC}\left(\Omega_{q}^{\mathrm{NS}}=0\right)$ and (b) QKS with $\Omega_{q}^{\mathrm{NS}}=10^{-15}$, letting $f_{a}$ vary in the interval $\left(10^{10}-10^{12}\right) \mathrm{GeV}$. The selected $\Omega_{q}^{\mathrm{NS}}$ is the largest possible value that ensures the validity of HTLA, since $T_{\mathrm{KR}} \simeq 10^{4} \mathrm{GeV}$. The black solid [dashed] lines correspond to the upper [lower] bound on $\Omega_{\tilde{a}} h^{2}$ in Eq. (1.2a), whereas the gray solid lines have been obtained by fixing $\Omega_{\tilde{a}} h^{2}$ to its central value in Eq. (1.2a) for $f_{a}=10^{11} \mathrm{GeV}$. In practice, the solid [dashed] line is constructed for $f_{a}=10^{12} \mathrm{GeV}\left[f_{a}=10^{10} \mathrm{GeV}\right]$. This is because $\Omega_{\tilde{a}} h^{2} \propto T_{\mathrm{I}} m_{\tilde{a}} / f_{a}^{2}\left[\Omega_{\tilde{a}} h^{2} \propto \ln T_{\mathrm{I}} m_{\tilde{a}} / f_{a}^{2}\right]$ in the SC [QKS] as deduced by Eqs. (3.24) and (3.4).

The left boundary curve (dot-dashed line) in Fig. 6-(a) comes from the lower bound of Eq. (1.2b). On the other hand, the upper boundary curve (double dot-dashed line) in Fig. 6-(b) comes from the upper bound on $T_{\mathrm{I}}, T_{\mathrm{I}} \leq f_{a}$. The lower boundary curves (dotted lines) in both Fig. 6-(a) and Fig. 6-(b) come from the saturation of $g_{3}>1$ which determines the range of validity of the HTLA. Needless to say that, for $T_{\mathrm{I}}<10^{4} \mathrm{GeV}$, imposing Eq. (2.13) also fails, since $T_{\mathrm{I}} \simeq T_{\mathrm{KR}}$.

We observe that in the SC the lower bound of Eq. (1.2b) is more restrictive than our requirement $T_{\mathrm{I}}<T_{\mathrm{D}}^{\mathrm{SC}}$, which is satisfied for all $f_{a}$ 's - see Eqs. (5.2) and (5.3). On the other hand, in the QKS, the selected $\Omega_{q}^{\mathrm{NS}}$ fulfills Eq. (5.5). For central values of $f_{a}$ and $\Omega_{\tilde{a}} h^{2}$ we find $1.85 \times 10^{7} \lesssim T_{\mathrm{I}} / \mathrm{GeV} \lesssim 10^{4}$ for $10^{-5} \lesssim m_{\tilde{a}} / \mathrm{GeV} \lesssim 0.018$ in the $\mathrm{SC}$, whilst $10^{11} \lesssim T_{\mathrm{I}} / \mathrm{GeV} \lesssim 10^{4}$ for $0.0015 \lesssim m_{\tilde{a}} / \mathrm{GeV} \lesssim 0.02$ in the QKS. We observe that in the SC the allowed $T_{\mathrm{I}}$ and $m_{\tilde{a}}$ are rather low, whereas in the QKS larger $T_{\mathrm{I}}$ and $m_{\tilde{a}}$ are permitted. Note, also, that towards lower $T_{\mathrm{I}}$ the preferred $m_{\tilde{a}}$ tends to larger values, implying that the LTR is cosmologically interesting in the case of $\tilde{a}$-CDM (contrary to the case of $\widetilde{G}-\mathrm{CDM})$.

\subsection{The Low $T$ Regime}

In this regime $\Omega_{\tilde{a}} h^{2}$ includes sizeable contributions from thermal scatterings and decay of SUSY partners. As a consequence, our computation depends not only on the parameters of the previous case $\left(m_{\tilde{a}}, f_{a}, T_{\mathrm{I}}\right.$ and $\left.\Omega_{q}^{\mathrm{NS}}\right)$, but also on the masses of the SUSY particles, $m_{i}$. We use the $m_{i}$ 's of 


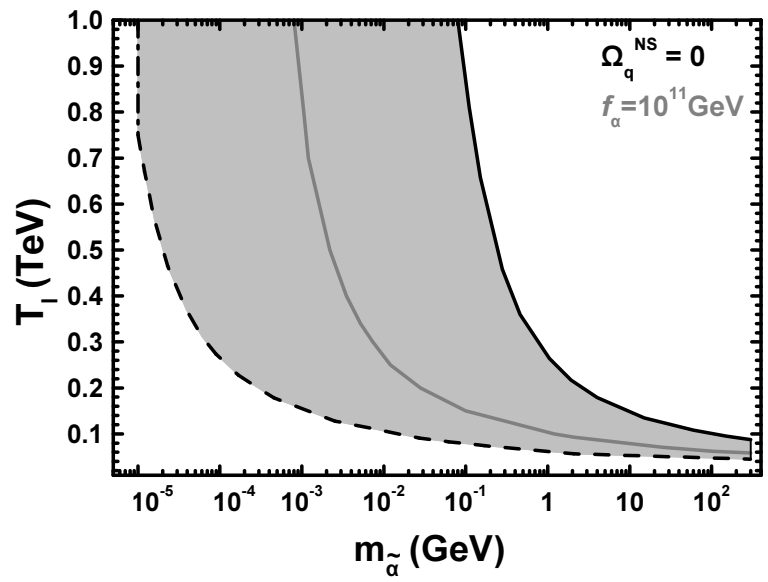

(a)

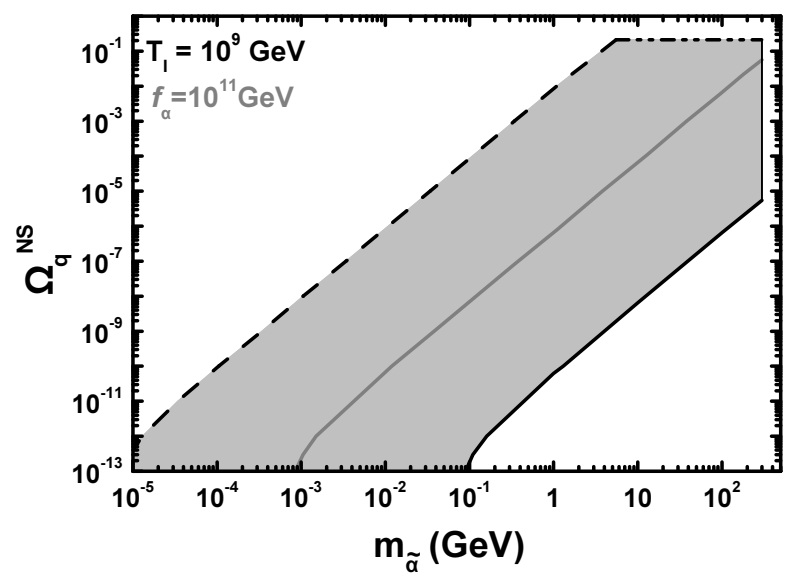

(b)

Figure 7: Allowed (lightly gray shaded) regions for $\tilde{a}-C D M, 10^{10} \leq f_{a} / \mathrm{GeV} \leq 10^{12}$ and $m_{i}$ 's shown in Eq. (3.29) in the LTR and (a) in the $m_{\tilde{a}}-T_{\mathrm{I}}$ plane for the $S C\left(\Omega_{q}^{\mathrm{NS}}=0\right)$ and (b) in the $m_{\tilde{a}}-\Omega_{q}^{\mathrm{NS}}$ plane for the $Q K S$ with $T_{\mathrm{I}}=10^{9} \mathrm{GeV}$. The conventions adopted for the various lines are also shown.

Eq. (3.29) for both the SC and the QKS. Needless to say that our preliminary assumptions $T_{\mathrm{I}}<f_{a}$ and $T_{\mathrm{I}}<T_{\mathrm{D}}^{\mathrm{SC}}\left[T_{\mathrm{KR}}<T_{\mathrm{D}}^{\mathrm{SC}}\right]$ are comfortably satisfied in the $\mathrm{SC}[\mathrm{QKS}]$.

In the $\mathrm{SC}\left(\Omega_{q}^{\mathrm{NS}}=0\right)$, the allowed (lightly gray shaded) region for $\tilde{a}$-CDM is shown in the $m_{\tilde{a}}-T_{\mathrm{I}}$ plane - see Fig. 7-(a). On the other hand, in the QKS, our results are independent of $T_{\mathrm{I}}-$ see Eq. (3.31); consequently, we depict the allowed (lightly gray shaded) region in the $m_{\tilde{a}}-\Omega_{q}^{\mathrm{NS}}$ plane with fixed $T_{\mathrm{I}}=10^{9} \mathrm{GeV}-$ see Fig. 7-(b). The black solid [dashed] line corresponds to the upper [lower] bound on $\Omega_{\tilde{a}} h^{2}$ in Eq. (1.2a), whereas the gray solid lines have been obtained by fixing $\Omega_{\tilde{a}} h^{2}$ to its central value in Eq. (1.2a) for $f_{a}=10^{11} \mathrm{GeV}$. In practice, the solid [dashed] line is constructed for $f_{a}=10^{12} \mathrm{GeV}$ $\left[f_{a}=10^{10} \mathrm{GeV}\right]$. This is because $T_{\mathrm{I}} \propto B \ln \left(f_{a}^{2} \Omega_{\tilde{a}} h^{2}\right)-B \ln \left(A m_{\tilde{a}}\right)\left[\sqrt{\Omega_{q}^{\mathrm{NS}}} \propto m_{\tilde{a}} / \Omega_{\tilde{a}} h^{2} f_{a}^{2}\right]$ in the SC [QKS] as deduced by Eq. (3.30) [Eq. (3.31)].

In the SC, the lower bound of Eq. (1.2b) determines a part (dot-dashed line) of the left boundary curve in Fig. 6-(a), whereas the upper bound of Eq. (1.2b) sets an upper bound (thin lines) in both Fig. 6-(a) and Fig. 6-(b). The upper boundary curve (double dot-dashed line) in Fig. 6-(b) comes from the upper bound on $\Omega_{q}^{\mathrm{NS}}$ in Eq. (2.14). The allowed area in Fig. 6-(a) [Fig. 6-(b)] terminates from above [below] at $T_{\mathrm{I}} \simeq 1 \mathrm{TeV}\left[T_{\mathrm{KR}} \simeq 1 \mathrm{TeV}\right.$ ], so that our formulas for $C_{\tilde{a}}^{\mathrm{LT}}$ to be fully applicable.

A sharp suppression of $\Omega_{\tilde{a}} h^{2}$ is observed, due to the Boltzmann suppression factor $e^{-B / T_{\mathrm{I}}}$, in the $\mathrm{SC}-$ see Fig. 7-(a). In this case, larger $m_{\tilde{a}}$ but very low $T_{\mathrm{I}}$ are allowed. On the contrary, $T_{\mathrm{I}}$ can be fixed to a naturally [58] high value within the QKS. At the same time, $m_{\tilde{a}}$ and $\Omega_{q}^{\mathrm{NS}}$ take interestingly large values in the allowed region - see Fig. 7-(b). For these reasons, this case is considered as the most intriguing of this paper. For central values of $f_{a}$ and $\Omega_{\tilde{a}} h^{2}$ we find $58.5 \lesssim T_{\mathrm{I}} / \mathrm{GeV} \lesssim 1000$ for $300 \gtrsim m_{\tilde{a}} / \mathrm{GeV} \gtrsim 8 \times 10^{-4}$ in the SC, whereas $10^{-13} \lesssim \Omega_{q}^{\mathrm{NS}} \lesssim 0.056$ for $8.5 \times 10^{-4} \lesssim m_{\tilde{a}} / \mathrm{GeV} \lesssim 300$ in the QKS $\left(T_{\mathrm{I}}=10^{9} \mathrm{GeV}\right)$. Our formalism of Appendix A is of crucial importance in order to obtain a reliable result in both the SC and QKS.

Finally, it would be interesting to directly compare the naturality of the $\tilde{a}$ and $\widetilde{G}$ as CDM candidates in the QKS. This is done in Fig. 8 where, contrary to our strategy in Fig. 5-(b) [Fig. 7(b)], we fix $m_{\widetilde{G}}\left[m_{\tilde{a}}\right]$ to some exemplary value and let $T_{\mathrm{I}}$ and $\Omega_{q}^{\mathrm{NS}}$ vary in their allowed region of 


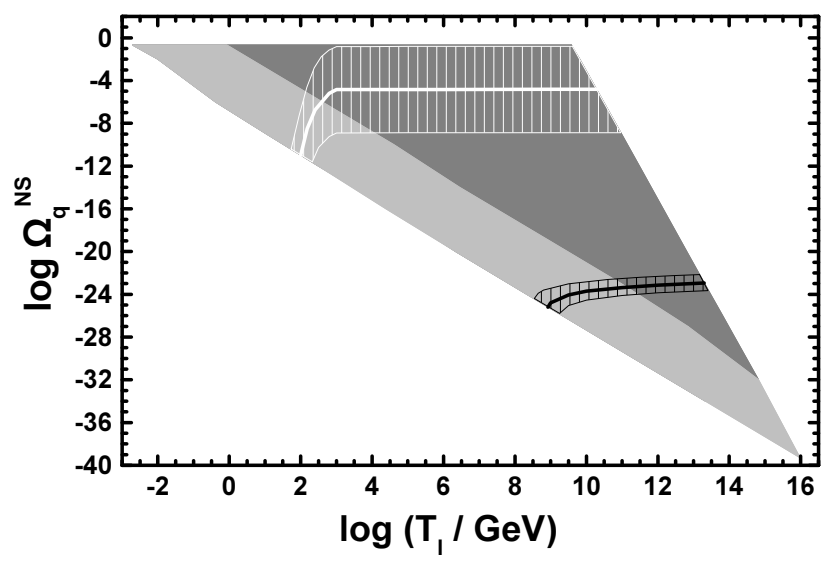

Figure 8: Simultaneously allowed areas in the $\log T_{\mathrm{I}}-\log \Omega_{q}^{\mathrm{NS}}$ plane taking into account the quintessential requirements (gray and lightly gray shaded area) - Eqs. (2.13), (2.14) and (2.15) - and the CDM constraint for $\widetilde{G}-C D M$ (black lined area) with $m_{\widetilde{G}}=100 \mathrm{GeV}$ and $0.5 \leq M_{1 / 2} / \mathrm{TeV} \leq 1$ or $\tilde{a}$-CDM (white lined area) with $m_{\tilde{a}}=5 \mathrm{GeV}, m_{i}$ 's of Eq. (3.29) and $10^{10} \leq f_{a} / \mathrm{GeV} \leq 10^{12}$ (white lined area). The bold black [white] line is obtained for $M_{1 / 2}=0.7 \mathrm{TeV}\left[f_{a}=10^{11} \mathrm{GeV}\right]$ and corresponds to $\Omega_{X} h^{2} \simeq 0.11$ (with $X=\widetilde{G}[X=\tilde{a}]$ ).

Fig. 2. In particular, in Fig. 8 we present in the $\log T_{\mathrm{I}}-\log \Omega_{q}^{\mathrm{NS}}$ plane the allowed region by both the quintessential requirements and the CDM constraint for $\widetilde{G}$-CDM or $\tilde{a}$-CDM. The first set of constraints - Eqs. (2.13)-(2.15) - is satisfied in the gray and lightly gray shaded area. The CDM constraint Eq. (1.2) - for $\widetilde{G}$-CDM with $m_{\widetilde{G}}=100 \mathrm{GeV}$ and $0.5 \leq M_{1 / 2} / \mathrm{TeV} \leq 1$ is fulfilled in the black lined region, where $\Omega_{q}^{\mathrm{NS}}$ is tuned to low values. The upper [lower] boundary curve of the black lined region corresponds to $\Omega_{\widetilde{G}} h^{2}=0.097\left[\Omega_{\widetilde{G}} h^{2}=0.12\right]$ and is constructed for $M_{1 / 2}=1 \mathrm{TeV}$ $\left[M_{1 / 2}=0.5 \mathrm{TeV}\right]$. This is because $\sqrt{\Omega_{q}^{\mathrm{NS}}} \propto M_{\alpha}^{2} / m_{\widetilde{G}} \Omega_{\widetilde{G}} h^{2}$ as concluded by Eqs. (3.4) and (3.24). On the contrary, Eq. (1.2) for $\tilde{a}$-CDM with $m_{\tilde{a}}=5 \mathrm{GeV}$ and $10^{10} \leq f_{a} / \mathrm{GeV} \leq 10^{12}$ is met in the white lined region, with much more natural $\Omega_{q}^{\mathrm{NS}}$ 's than the ones needed in the black lined region. For the required $\Omega_{q}^{\mathrm{NS}}$ 's our results of the LTR are applicable. For $T_{\mathrm{I}} \gtrsim 1 \mathrm{TeV}, \Omega_{\tilde{a}} h^{2}$ is obviously independent on $T_{\mathrm{I}}$ and Eq. (3.31) approaches fairly our numerical results. The upper [lower] boundary curve of the white lined region corresponds to $\Omega_{\tilde{a}} h^{2}=0.097\left[\Omega_{\tilde{a}} h^{2}=0.12\right]$ and is constructed for $f_{a}=10^{10} \mathrm{GeV}\left[f_{a}=10^{12} \mathrm{GeV}\right]$ (in accordance with our discussion above). The bold black [white] lines are constructed for $M_{1 / 2}=0.7 \mathrm{TeV}\left[f_{a}=10^{11} \mathrm{GeV}\right]$ and correspond to $\Omega_{X} h^{2} \simeq 0.11$. For these values, we find $8 \times 10^{8} \lesssim T_{\mathrm{I}} / \mathrm{GeV} \lesssim 2 \times 10^{13}$ and $6.3 \times 10^{-26} \lesssim \Omega_{q}^{\mathrm{NS}} \lesssim 10^{-23}$ for $\widetilde{G}$-CDM, whereas $92.2 \lesssim T_{\mathrm{I}} / \mathrm{GeV} \lesssim 1.8 \times 10^{10}$ and $1.25 \times 10^{-11} \lesssim \Omega_{q}^{\mathrm{NS}} \lesssim 1.6 \times 10^{-5}$ for $\tilde{a}$-CDM. Therefore, in the framework of the QKS, $\tilde{a}$ is clearly a more natural CDM candidate than $\widetilde{G}$.

\section{Conclusions}

We presented an exponential quintessential model which generates a period dominated by the kinetic energy of the quintessence field. The parameters of the quintessential model $\left(\lambda, T_{\mathrm{I}}, \Omega_{q}^{\mathrm{NS}}\right)$ were confined so that $0.5 \leq \Omega_{q}\left(T_{\mathrm{I}}\right) \leq 1$, and were constrained by current observational data originating from NS, the acceleration of the universe, the inflationary scale and the DE density parameter. We found $0<\lambda<0.9$ and studied the allowed region in the $\left(T_{\mathrm{I}}, \Omega_{q}^{\mathrm{NS}}\right)$-plane.

We proceeded to examine the impact of this KD epoch to the thermal abundance of $\widetilde{G}$ and $\tilde{a}$. We solved the problem (i) numerically, integrating the relevant system of the differential equations equation 
that governs the evolution of the $X$-number density and (ii) semi-analytically, producing approximate relations for the cosmological evolution before and after the transition from KD to RD, and solving the appropriately re-formulated Boltzmann. Although we did not succeed to achieve general analytical solutions in all cases, we consider as a significant development the derivation of a result by solving numerically just one equation, instead of the whole system above. Moreover, for typical values of $m_{i}$ 's in Eq. (3.29), empirical formulas that reproduce quite successfully our numerical results were derived. For unstable $\widetilde{G}$, the $\widetilde{G}$-constraint poses a lower bound on $\Omega_{q}^{\mathrm{NS}}$, which turns out to be almost independent of $T_{\mathrm{I}}$. The CDM constraint can be satisfied by the thermal abundance of $\widetilde{G}$ for extremely low values $\left(10^{-23}-10^{-24}\right)$ of $\Omega_{q}^{\mathrm{NS}}$. On the contrary, the former constraint can be fulfilled by the thermal abundance of $\tilde{a}$ with values of $\Omega_{q}^{\mathrm{NS}}$ close to the upper bound posed by the requirement for successful NS.

Let us also comment here on three minor subtleties of our calculation which, do not alter the basic features of our conclusions (although could potentially create some quantitative modifications to our results). In particular:

- Throughout our investigation we did not identify the nature of NLSP. Therefore the upper bound, shown in Fig. 5-(a) [Fig. 7], on $m_{\widetilde{G}}\left[m_{\tilde{a}}\right]$ derived from the requirement $\left[m_{\widetilde{G}} \leq m_{\tilde{B}}\right] m_{\tilde{a}} \leq m_{\tilde{B}}$ could be modified if there is another SUSY particle lighter than $\tilde{B}$. Moreover, we did not consider the NS constraints concerning the late decays of the NLSP into $X$ 's. These constraints $[26,30,31]$ depend very much on the properties of the NLSP, i.e. its composition, its mass relative to the $m_{X}$, and its coupling to $X$ 's. Consequently, additional bounds on the $m_{X}$ might arise. As the $\tilde{a}$ interactions are not as strongly suppressed as the $\widetilde{G}$ interactions, the $\tilde{a}$ LSP anyhow is far less problematic than the $\widetilde{G}$ LSP w.r.t these constraints.

- In the case of $\widetilde{G}$, we did not incorporate contributions to $\Omega_{\widetilde{G}} h^{2}$ from the process of reheating. Indeed, these extra contributions can be a fraction of the result shown in Eq. (3.24) [34, 62], in the case of the usual reheating realized by the coherent oscillations of a massive particle [61]. However, in the QKS several reheating processes have been proposed [63] and therefore, any safe comparison between the SC and the QKS has to be performed for $T<T_{\mathrm{I}}$. In other words, to keep our investigation as general as possible, we preferred to study the evolution of the universe after the start of the RD [KD] era in the SC [QKS] (we simply assumed the existence of an earlier inflationary epoch).

- In the case of the $\tilde{a}$-CDM, we used throughout our investigation some representative masses for the superpartners, given in Eq. (3.29). Variation in these values (especially in $m_{\tilde{g}}$ and $m_{\tilde{q}}$ ) has an impact on $\Omega_{\tilde{a}} h^{2}$ in the LTR (e.g., for $m_{\tilde{g}} \simeq m_{\tilde{q}}$ the contribution of the process $\tilde{q}^{*} q$ to $C_{\tilde{a}}^{\mathrm{LT}}$ can be enhanced). In addition, a further uncertainty in our calculation arises from the determination of $T_{\mathrm{SUSY}}$ below which $C_{\tilde{a}}^{\mathrm{HT}}$ is replaced by $C_{\tilde{a}}^{\mathrm{LT}}$ in the integration of the relevant equations. Note however, that the aim of the present paper is to demonstrate the change of $\Omega_{X} h^{2}$ due to the presence of the KD era and not a full scan of the SUSY parameter space.

Although our results have been derived in the context of an exponential quintessential model, their applicability can be extended to every model that generates a KD phase, even without [6] quintessential consequences. It is worth mentioning that in the presence of kination we can obtain simultaneous compatibility of both the gravitino and the CDM constraint (i.e., the lower bound on $\Omega_{q}^{\mathrm{NS}}$ from the gravitino constraint is compatible with the $\Omega_{q}^{\mathrm{NS}}$, s needed in order to have the correct amount of $\tilde{a}$ $\mathrm{CDM})$. It would be probably interesting to check if we can obtain a simultaneous compatibility of these two constraints with additional bounds, arising e.g. from leptogenesis and neutrino masses $[64,65]$ or the quintessino abundance [66]. 
Quintessential Kination and Thermal Production of Gravitinos and Axinos

\section{ACKNOWLedgments}

We would like to thank K.Y. Choi, R. Ruiz de Austri and L. Roszkowski for helpful discussions. The research of S.L was funded by the FP6 Marie Curie Excellence Grant MEXT-CT-2004-014297. The work of M.E.G, C.P and J.R.Q was supported by the Spanish MEC project FPA2006-13825 and the project P07FQM02962 funded by the "Junta de Andalucia".

\section{A. Axino Production via Scatterings at Low Temperature}

In this appendix we present the necessary ingredients used for the evaluation of the $\tilde{a}$-production via scatterings at low $T$. Our starting point is the general formula in Ref. [67], which gives the thermal averaged cross section times the relativistic invariant relative velocity, $\left\langle(v \sigma)_{i j}\right\rangle$, of two particles $i$ and $j$ with masses $m_{i}$ and $m_{j}$ (in general $m_{i} \neq m_{j}$ ) and degrees of freedom $g_{i}$ and $g_{j}$ respectively:

$$
\left\langle(v \sigma)_{i j}\right\rangle=\frac{1}{2 m_{i}^{2} m_{j}^{2} T K_{2}\left(\frac{m_{i}}{T}\right) K_{2}\left(\frac{m_{j}}{T}\right)} \int_{s_{0}}^{\infty} d s w_{i j}(s) K_{1}\left(\frac{\sqrt{s}}{T}\right) p_{\mathrm{i}}\left(m_{i}, m_{j}\right)
$$

where $s_{0}=\left(m_{i}+m_{j}\right)^{2}$ and

$$
w_{i j}(s)=\frac{g_{i} g_{j}}{32 \pi} \frac{p_{\mathrm{f}}\left(m_{k}, m_{\tilde{a}}\right)}{\sqrt{s}} \int_{-1}^{+1} d \cos \theta_{\mathrm{CM}}\left|\mathcal{M}_{i j}\right|^{2} .
$$

Here, $\left|\mathcal{M}_{i j}\right|^{2}$ is the squared amplitude of the process $i+j \rightarrow k+\tilde{a}$ which contributes to $\left\langle(v \sigma)_{i j}\right\rangle, m_{k}$ is the mass of the outgoing particle $k$ in this process, $\theta_{\mathrm{CM}}$ is the scattering angle in the center-of-mass frame and $p_{\mathrm{i}}$ and $p_{\mathrm{f}}$ are the magnitudes of the incoming and outcoming 3-momentums in the same reference frame; these are given respectively by:

$$
\begin{aligned}
& p_{\mathrm{i}}\left(m_{i}, m_{j}\right)=\frac{\lambda\left(m_{i}, m_{j}\right)}{\sqrt{s}} \text { and } p_{\mathrm{f}}\left(m_{k}, m_{\tilde{a}}\right)=\frac{\lambda\left(m_{k}, m_{\tilde{a}}\right)}{\sqrt{s}} \\
& \text { where } \lambda^{2}\left(m_{i}, m_{k}\right)=\frac{1}{4}\left(s-\left(m_{i}+m_{k}\right)^{2}\right)\left(s-\left(m_{i}-m_{k}\right)^{2}\right)
\end{aligned}
$$

Multiplying $\left\langle(v \sigma)_{i j}\right\rangle$ by $n_{i}^{\mathrm{eq}} n_{j}^{\mathrm{eq}}$ and using Eq. (3.2) we arrive at our final result in Eq. (3.3), with $C_{X}=C_{\tilde{a}}^{\mathrm{LT}}$ given by Eq. (3.8).

For the manipulation of this result it would be useful to remember that $\left|\mathcal{M}_{i j}\right|^{2}$ is in general a function of the Mandelstam variables, $s, t$ and $u$ (and the masses of the involved particles). However, one of the Mandelstam variables, typically $u$, can be eliminated in favor of the other two, through the formula:

$$
u=m_{i}^{2}+m_{j}^{2}+m_{k}^{2}+m_{\tilde{a}}^{2}-s-t
$$

whereas in the reference frame of the center of mass, $t$ can be expressed in terms of $s$ and $\theta_{\mathrm{CM}}$ using Eq. (A5) and the relation [67]:

$$
t-u=-\frac{\left(m_{i}^{2}-m_{j}^{2}\right)\left(m_{k}^{2}-m_{\tilde{a}}^{2}\right)}{s}+4 p_{\mathrm{i}}(s) p_{\mathrm{f}}(s) \cos \theta_{\mathrm{CM}}
$$

Therefore, $\left|\mathcal{M}_{i j}\right|^{2}$ can be written as a function of only $s$ and $\theta_{\mathrm{CM}}$.

The computation of the various $\left|\mathcal{M}_{i j}\right|^{2}$ can be realized applying standard techniques [68]. We concentrate on the $\tilde{a}$ production processes which involve $S U(3)_{\mathrm{C}}$ interactions. The Feynman rules for these interactions are indicated in Fig. 9 and originate from the following Lagrangian term [26, 27]:

$$
\mathcal{L}_{\tilde{a} \tilde{g} g}=i \frac{g_{a}}{2} \overline{\tilde{a}} \gamma_{5}\left[\gamma^{\mu}, \gamma^{\nu}\right] \tilde{g}^{\mathrm{a}} G_{\mu \nu}^{\mathrm{a}} \text {, where } g_{a}=\frac{g_{3}^{2}}{32 \pi^{2} f_{a}},
$$



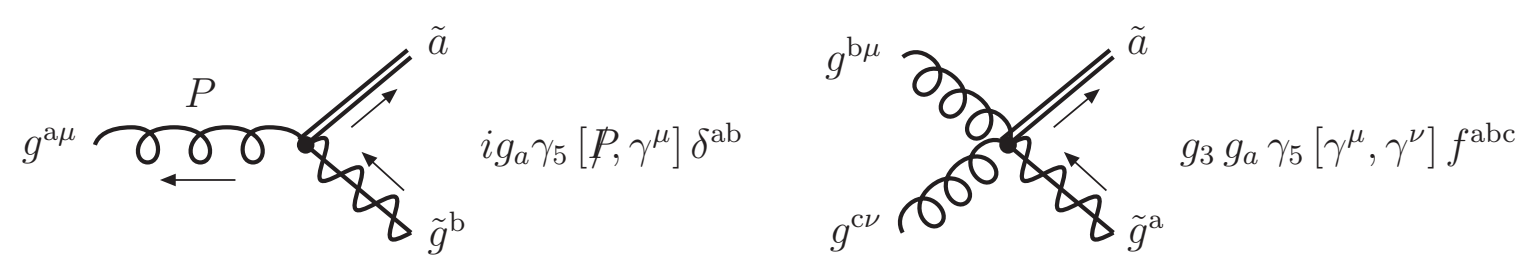

Figure 9: Feynman rules used in the derivation of $\left|\mathcal{M}_{i j}\right|^{2}$ for the vertices with $\tilde{a}$. The arrows were drawn according to the conventions of Ref. [68].

$G_{\mu \nu}^{\mathrm{a}}=\partial_{\mu} g_{\nu}^{\mathrm{a}}-\partial_{\nu} g_{\mu}^{\mathrm{a}}-g_{3} f^{\mathrm{abc}} g_{\mu}^{\mathrm{b}} g_{\mu}^{\mathrm{c}}$ is the gluon field tensor, $f^{\mathrm{abc}}$ are the structure constants of the $S U(3)_{\mathrm{C}}$ algebra with $\mathrm{a}, \mathrm{b}, \mathrm{c}=1, \ldots, 8$ whereas $\mu$ and $\nu$ are the usual spacetime indices. The residual Feynman rules needed for our calculation are taken from Ref. [69]. In Table 1, we list the Feynman diagrams included in the calculation of $\left|\mathcal{M}_{i j}\right|^{2}$. Note that in the non-relativistic regime some of the processes $(g g$, $q \bar{q}$ and $g q$ ) contributing to $\left|\mathcal{M}_{i j}\right|^{2}$ in the relativistic regime [27] are kinematically blocked and thus, are non included in our computation.

In Table 2 we list analytical expressions for the non-averaged squared amplitudes $g_{i} g_{j}\left|\mathcal{M}_{i j}\right|^{2}$ 's of the various scatterings of $i j$ particles. Let us make some comments on the findings that we present in this table:

- Although our numerical results for the $m_{i}$ 's of Eq. (3.29) are practically independent on $m_{\tilde{a}}$ (since it is expected to be significantly lower than $m_{i}$ 's - especially $m_{\tilde{g}}$ and $m_{\tilde{q}}$ ), we explicitly display it in our formulas. Only gluons and quarks are taken to be massless. Due to the smallness of $m_{\tilde{a}}$ as compared to $m_{i}$ 's, $C_{\tilde{a}}^{\mathrm{LT}}$ can be approximated by an interpolated function of $T$, for every chosen $m_{i}$ 's. This is, in practice, a great simplification for our numerical treatment.

- The Feynman gauge has been used throughout our computation. The substraction of the unphysical mode in the process $g \tilde{g}$ with two external $g$ 's can be obtained by employing appropriate projection operators [70] for the transverse polarization states.

- We present the complete expressions for all $g_{i} g_{j}\left|\mathcal{M}_{i j}\right|^{2}$,s, except for the case of $g \tilde{g}$, where $g_{i} g_{j}\left|\mathcal{M}_{i j}\right|^{2}$ turns out to be very lengthy; in this latter case, we display only the important contributions which arise from the t-channel $\tilde{g}$ exchange and the interference terms between the $t$ and $s$ channel and PI. We have checked that our results on the total squared matrix elements for $\tilde{a}$ production agree nicely with those of Ref. [27] in the relativistic limit (setting $m_{\tilde{g}}=m_{\tilde{q}}=m_{\tilde{a}}=0$ ).

- In the expressions we present, $g_{i} g_{j}\left|\mathcal{M}_{i j}\right|^{2}$ s are weighted with appropriate multiplicities following Ref. [69]. In particular, in the second column of Table 2 we arrange factors due to the summation over all the color-triplets $\left(N_{\mathrm{F}}=12\right)$ - taking into account the corresponding charge conjugate multiplets which imply an extra factor of 2 . The factor $1 / 2$ occurs in the $\tilde{g} \tilde{g}$ process because of the identical particles in the initial state. Factors arising from the summation over color degrees of freedom and color indices, according to the identities

$$
\sum_{\mathrm{a}, \mathrm{b}, \mathrm{c}}\left|f^{\mathrm{abc}}\right|^{2}=N_{3}\left(N_{3}^{2}-1\right) \text { and } \sum_{a, I, J}\left|T_{I J}^{\mathrm{a}}\right|^{2}=\frac{1}{2}\left(N_{3}^{2}-1\right),
$$

are included clearly in the expressions of the third column $\left(T_{I J}^{\mathrm{a}}\right.$ are the generators of the $S U(3)_{\mathrm{C}}$ group and $N_{3}=3$ ).

- The decay width of $\tilde{g}$ is evaluated employing the tree level result of Ref. [71] - possible next-toleading order corrections are subdominant. The result is checked to ensure that it is consistent 


\begin{tabular}{|c|c|c|}
\hline Initial & FinaL & $\begin{array}{c}\text { INTERACTION } \\
\text { ChanNels }\end{array}$ \\
StATE & StATE & $s\left(g^{\mathrm{c}}\right), t\left(g^{\mathrm{b}}\right), u\left(g^{\mathrm{a}}\right)$ \\
\hline$\tilde{g}^{\mathrm{a}} \tilde{g}^{\mathrm{b}}$ & $g^{\mathrm{c}} \tilde{a}$ & $t\left(g^{\mathrm{a}}\right)$ \\
$\tilde{q}_{I} \tilde{g}^{\mathrm{a}}$ & $\tilde{q}_{J} \tilde{a}$ & $s\left(\tilde{g}^{\mathrm{c}}\right), t\left(g^{\mathrm{b}}\right), u\left(\tilde{g}^{\mathrm{a}}\right)$, PI \\
$g^{\mathrm{a}} \tilde{g}^{\mathrm{b}}$ & $\tilde{g}^{\mathrm{c}} \tilde{a}$ & $t\left(g^{\mathrm{a}}\right)$ \\
$q_{I} \tilde{g}^{\mathrm{a}}$ & $q_{J} \tilde{a}$ & $s\left(g^{\mathrm{a}}\right)$ \\
$\tilde{q}_{I} \tilde{q}_{J}^{*}$ & $\tilde{g}^{\mathrm{a}} \tilde{a}$ & $t\left(\tilde{g}^{\mathrm{a}}\right)$ \\
$\tilde{q}_{I} g^{\mathrm{a}}$ & $q_{J} \tilde{a}$ & $s\left(\tilde{g}^{\mathrm{a}}\right)$ \\
$\tilde{q}_{I}^{*} q_{J}$ & $g^{\mathrm{a}} \tilde{a}$ & \\
\hline
\end{tabular}

Table 1: The Feynman diagrams contributing to $\left\langle(v \sigma)_{i j}\right\rangle$ with $\tilde{a}$ in the final state. The exchanged particles are indicated for each relevant pair of initial and final states. The symbols $s(i), t(i)$ and $u(i)$ denote treegraphs in which the particle $i$ is exchanged in the s-, $t$ - or $u$-channel and PI stands for "point interaction". The superscripts are $S U(3)$ color indices, whereas the subscript $I$ and $J$ are family indices.

with the output of the calcHEP package [72], and is the following:

$$
\Gamma_{\tilde{g}}=2 N_{\mathrm{F}} \frac{g_{3}(T)}{32 \pi} m_{\tilde{g}}\left(1-\left(\frac{m_{\tilde{q}}}{m_{\tilde{g}}}\right)^{2}\right)^{2} .
$$

- Although some integrals in Eq. (3.8) (especially the ones corresponding to the processes $g \tilde{g}$ and $q \tilde{g})$ turn out to converge slowly, we checked that our results are pretty stable without the need to introduce an effective mass for the gluons propagators, as in the relativistic case [24, 26, 27].

- In our numerical computation and with the $m_{i}$ 's listed in Eq. (3.29), we do not include the contributions of the processes $\tilde{q} \tilde{q}^{*}$ and $\tilde{q} g$ since they are, in general, negligible. The major contributions to $\left|\mathcal{M}_{i j}\right|^{2}$ come from $\tilde{g} \tilde{g}$ and $\tilde{q} \tilde{g}$ for $T \simeq T_{\text {SUSY }}$ and from $\tilde{q} q$ for $T \ll T_{\text {SUSY. The }}$ processes $g \tilde{g}$ and $q \tilde{g}$ give in general important contributions which cannot be neglected. Note, however, that the contribution of the process $\tilde{q}^{*} q$ could also be enhanced, if $m_{\tilde{g}} \simeq m_{\tilde{q}}$, since we would have a resonance in the $\tilde{q}-q$ annihilation via an $s$-channel exchange of a $\tilde{g}$. However, we consider that the choice $m_{\tilde{g}} \simeq m_{\tilde{q}}$ would be a rather ugly tuning of our free parameters and thus, we opted to use the $m_{i}$ 's in Eq. (3.29) in order to demonstrate our findings.

\section{REFERENCES}

[1] D.N. Spergel et al. [WMAP Collaboration], Astrophys. J. Suppl. 170, 377 (2007) [astro-ph/0603449]; E. Komatsu et al.[WMAP Collaboration], arXiv:0803.0547.

[2] M. Tegmark et al. [SDSS Collaboration], Phys. Rev. D 69, 103501 (2004) [astro-ph/0310723]; A.G. Riess et al. [SST Collaboration], Astrophys. J.607, 665 (2004) [astro-ph/0402512].

[3] For a review from the viewpoint of particle physics, see A.B. Lahanas et al., Int. J. Mod. Phys. D 12, 1529 (2003) [hep-ph/0308251].

[4] R.R. Caldwell et al., Phys. Rev. Lett. 80, 1582 (1998) [astro-ph/9708069].

[5] P. Binetrui, Int. J. Theor. Phys. 39, 1859 (2000) [hep-ph/0005037]; E.J. Copeland et al., Int. J. Mod. Phys. D 15, 1936 (2006) [hep-th/0603057].

[6] B. Spокоiny, Phys. Lett. B 315, 40 (1993) [gr-qc/9306008]; M. Joyce, Phys. Rev. D 55, 1875 (1997) [hep-ph/9606223]; P.G. Ferreira and M. Joyce, Phys. Rev. D 58, 023503 (1998) [astro-ph/9711102].

[7] P.J. Peebles and A. Vilenkin, Phys. Rev. D 59, 063505 (1999) [astro-ph/9810509]; M. Peloso and F. Rosatı, J. High Energy Phys. 12, 026 (1999) [hep-ph/9908271]. 


\begin{tabular}{|c|c|c|}
\hline$i j$ & & $g_{i} g_{j}\left|\mathcal{M}_{i j}\right|^{2} / g_{a}^{2} g_{3}^{2}$ \\
\hline$\tilde{g} \tilde{g}$ & $\frac{1}{2}$ & $\begin{array}{l}\frac{32 N_{3}\left(N_{3}+1\right)}{(s t u)^{2}}\left[2 m_{\tilde{g}}^{2}\left(m_{\tilde{a}}^{2}-m_{\tilde{g}}^{2}\right)^{2} s^{2} t^{2}+s t\left(4 m_{\tilde{g}}^{3}\left(m_{\tilde{g}}-m_{\tilde{a}}\right)\left(m_{\tilde{g}}+m_{\tilde{a}}\right)^{2} s\right.\right. \\
-\left(m_{\tilde{g}}-m_{\tilde{a}}\right)\left(2 m_{\tilde{g}}^{2}\left(2 m_{\tilde{g}}-m_{\tilde{a}}\right)\left(m_{\tilde{g}}+m_{\tilde{a}}\right)^{2}+\left(m_{\tilde{g}}-m_{\tilde{a}}\right)\left(3 m_{\tilde{g}}^{2}+2 m_{\tilde{g}} m_{\tilde{a}}\right.\right. \\
\left.\left.\left.+m_{\tilde{a}}^{2}\right) s\right) t+2 s\left(3 m_{\tilde{g}}^{2}-2 m_{\tilde{g}} m_{\tilde{a}}+s\right) t^{2}\right) u-\left(2 m_{\tilde{g}}^{2}\left(m_{\tilde{g}}^{2}-m_{\tilde{a}}^{2}\right)^{2} s^{2}+s t\left(\left(7 m_{\tilde{g}}^{4}\right.\right.\right. \\
\left.\left.+m_{\tilde{a}}^{4}\right) s+2 m_{\tilde{g}}^{2}\left(m_{\tilde{g}}+m_{\tilde{a}}\right)^{2}\left(2 m_{\tilde{g}}^{2}-3 m_{\tilde{g}} m_{\tilde{a}}+m_{\tilde{a}}^{2}\right)\right)+\left(2 m_{\tilde{g}}^{2}\left(m_{\tilde{g}}^{2}-m_{\tilde{a}}^{2}\right)^{2}\right. \\
\left.\left.+\left(3 m_{\tilde{g}}^{4}+4 m_{\tilde{g}}^{3} m_{\tilde{a}}+m_{\tilde{a}}^{4}\right) s-\left(m_{\tilde{g}}-m_{\tilde{a}}\right)\left(5 m_{\tilde{g}}+3 m_{\tilde{a}}\right) s^{2}-2 s^{3}\right) t^{2}-2 s^{2} t^{3}\right) u^{2} \\
\left.+2 s t\left(-s t+3 m_{\tilde{g}}^{2}(s+t)+m_{\tilde{a}}^{2}(s+t)\right) u^{3}-2 s t(s+t) u^{4}\right]\end{array}$ \\
\hline$\tilde{q} \tilde{g}$ & $2 N_{\mathrm{F}}$ & $\begin{array}{l}\frac{4}{t^{2}}\left(N_{3}^{2}-1\right)\left(2 m_{\tilde{g}}^{4} m_{\tilde{a}}^{2} t\left(t-4 m_{\tilde{q}}^{2}\right)-4\left(m_{\tilde{g}}^{2}+m_{\tilde{a}}^{2}\right) m_{\tilde{q}}^{2}\right. \\
\left.-t\left(t+2 u-2 m_{\tilde{q}}^{2}\right)+m_{\tilde{a}}^{2} t(t+4 u)+m_{\tilde{g}}^{2}\left(4 m_{\tilde{a}}^{2}\left(2 m_{\tilde{q}}^{2}-t\right)+t(t+4 u)\right)\right)\end{array}$ \\
\hline$g \tilde{g}$ & 1 & $\begin{array}{l}N_{3}\left(N_{3}-1\right)\left[\frac{8\left(m_{\tilde{g}}^{2}-s\right)}{s^{2} t\left(\left(s-m_{\tilde{g}}^{2}\right)^{2}+\Gamma_{\tilde{g}}^{2} m_{\tilde{g}}^{2}\right)}\left(4 s m_{\tilde{g}}^{2}\left(m_{\tilde{g}}^{2}-m_{\tilde{a}}^{2}\right)\left(s-m_{\tilde{a}}^{2}\right)\left(m_{\tilde{g}}^{2}-2 m_{\tilde{a}}^{2}\right.\right.\right. \\
+s)+\left(m_{\tilde{g}}^{6}\left(m_{\tilde{a}}^{2}-2 s\right)-4 m_{\tilde{a}}^{4} s^{2}+m_{\tilde{g}}^{5}\left(6 m_{\tilde{a}}^{3}-4 m_{\tilde{a}} s\right)+m_{\tilde{g}}^{4}\left(m_{\tilde{a}}^{4}-21 m_{\tilde{a}}^{2} s+12 s^{2}\right)\right. \\
\left.-2 m_{\tilde{g}}^{3}\left(2 m_{\tilde{a}}^{5}+5 m_{\tilde{a}}^{3} s-6 m_{\tilde{a}} s^{2}\right)+m_{\tilde{g}}^{2}\left(6 s^{3}-2 m_{\tilde{a}}^{6}+27 m_{\tilde{a}}^{4} s-18 m_{\tilde{a}}^{2} s^{2}\right)\right) t \\
-\left(m_{\tilde{g}}^{2} m_{\tilde{a}}^{2}\left(3 m_{\tilde{g}}^{2}-6 m_{\tilde{g}} m_{\tilde{a}}-8 m_{\tilde{a}}^{2}\right)-\left(10 m_{\tilde{g}}^{4}+28 m_{\tilde{g}}^{3} m_{\tilde{a}}+m_{\tilde{g}}^{2} m_{\tilde{a}}^{2}-2 m_{\tilde{a}}^{4}\right) s+\right. \\
\left.\left.2\left(m_{\tilde{g}}+m_{\tilde{a}}\right)\left(3 m_{\tilde{g}}+m_{\tilde{a}}\right) s^{2}\right) t^{2}-2 t^{3}\left(2 m_{\tilde{g}} m_{\tilde{a}} s+2 m_{\tilde{g}}^{2}\left(m_{\tilde{a}}^{2}+2 s\right)-s\left(m_{\tilde{a}}^{2}+2 s\right)\right)\right) \\
+\frac{4}{s^{2} t}\left(16 m_{\tilde{g}}^{6} s+16 m_{\tilde{a}}^{6} s+2 m_{\tilde{g}}^{3} m_{\tilde{a}} t\left(8 t-3 m_{\tilde{a}}^{2}+12 s\right)-4 m_{\tilde{a}}^{4}\left(6 s^{2}+3 s t-2 t^{2}\right)\right. \\
+m_{\tilde{g}}^{4}\left(8 t^{2}-4\left(m_{\tilde{a}}^{4}+3 m_{\tilde{a}}^{2} s+6 s^{2}\right)+\left(m_{\tilde{a}}^{2}-12 s\right) t\right)+4 m_{\tilde{a}}^{2}\left(4 s^{3}+4 s^{2} t-5 s t^{2}\right. \\
\left.-4 t^{3}\right)-4(2 s+t)\left(2 s^{3}+3 s^{2} t-2 t^{3}\right)+8 t m_{\tilde{g}} m_{\tilde{a}}\left(m_{\tilde{a}}^{2}(3 s+2 t)-s^{2}-4 s t-2 t^{2}\right) \\
\left.\left.+m_{\tilde{g}}^{2}\left(m_{\tilde{a}}^{4}(t-12 s)+m_{\tilde{a}}^{2}\left(28 s^{2}+4 s t+19 t^{2}\right)+4\left(4 s^{3}+4 s^{2} t-5 s t^{2}-4 t^{3}\right)\right)\right)\right]\end{array}$ \\
\hline$q \tilde{g}$ & $2 N_{\mathrm{F}}$ & $8\left(N_{3}^{2}-1\right)\left(\left(m_{\tilde{g}}^{2}+m_{\tilde{a}}^{2}\right)(2 s+t)-m_{\tilde{g}}^{4}-m_{\tilde{a}}^{4}-2 m_{\tilde{g}} m_{\tilde{a}} t-2 s(s+t)\right) / t$ \\
\hline$\tilde{q} \tilde{q}^{*}$ & $N_{\mathrm{F}}$ & The same as for the process $\tilde{q} \tilde{g}$ but with the opposite sign and $s \leftrightarrow t$ \\
\hline$\tilde{q} g$ & $2 N_{\mathrm{F}}$ & $8\left(N_{3}^{2}-1\right)\left(t-m_{\tilde{a}}^{2}\right)\left(s t-m_{\tilde{a}}^{2} m_{\tilde{q}}^{2}+m_{\tilde{g}}^{2} u\right) /\left(t-m_{\tilde{g}}^{2}\right)^{2}$ \\
\hline$\tilde{q}^{*} q$ & $2 N_{\mathrm{F}}$ & $8\left(N_{3}^{2}-1\right)\left(s-m_{\tilde{a}}^{2}\right)\left(s t-m_{\tilde{a}}^{2} m_{\tilde{q}}^{2}+m_{\tilde{g}}^{2} u\right) /\left(\left(s-m_{\tilde{g}}^{2}\right)^{2}+\Gamma_{\tilde{g}}^{2} m_{\tilde{g}}^{2}\right)$ \\
\hline
\end{tabular}

Table 2: The quantities $g_{i} g_{j}\left|\mathcal{M}_{i j}\right|^{2}$ for a production from scatterings of the $i j$ particles in the nonrelativistic regime. The results are summed over spins, generation and color indices, in the initial and final state. The Mandelstam variables are defined as $s=\left(p_{1}+p_{2}\right)^{2}, t=\left(p_{1}-k_{1}\right)^{2}$ and $u=\left(p_{1}-k_{2}\right)^{2}$ where the four-momenta $p_{1}, p_{2}, k_{1}$, and $k_{2}$ are associated with the particles in the order in which they are written down in the columns "initial state" and "final state" of Table 1. 
[8] K. Dimopoulos and J.W. Valle, Astropart. Phys. 18, 287 (2002) [astro-ph/0111417];

K. Dimopoulos, Phys. Rev. D 68, 123506 (2003) [astro-ph/0212264].

[9] D.J.H. Chung, L.L. Everett and K.T. Matchev, Phys. Rev. D 76, 103530 (2007) [arXiv: 0704.3285];

G. Barenboim and J.D. Lykken, J. High Energy Phys. 10, 032 (2007) [arXiv: 0707 . 3999].

[10] M. Kamionkowski and M.S. Turner, Phys. Rev. D 3310, 42 (1990).

[11] P. Salati, Phys. Lett. B 571, 121 (2003) [astro-ph/0207396].

[12] S. Profumo and P. Ullio, J. Cosmol. Astropart. Phys. 11, 006 (2003) [hep-ph/0309220].

[13] C. Pallis, J. Cosmol. Astropart. Phys. 10, 015 (2005) [hep-ph/0503080].

[14] G. Barenboim and J.D. Lykken, J. High Energy Phys. 12, 005 (2006) [hep-ph/0608265]; D.J.H. Chung et al., J. High Energy Phys. 10, 016 (2007) [arXiv: 0706.2375].

[15] K. Matchev, hep-ph/0402088; E.A. Baltz, astro-ph/0412170; G. LaZARIDEs, hep-ph/0601016; M. Taoso, G. Bertone and A. Masiero, J. Cosmol. Astropart. Phys. 03, 022 (2008) [arXiv: 0711. 4996].

[16] H. Goldberg, Phys. Rev. Lett. 50, 1419 (1983); J.R. ElLis et al., Nucl. Phys. B238, 453 (1984).

[17] For other WIMPs in the context of the extra dimensional theories, see G. Servant and T.M.P. Tait, Nucl. Phys. B650, 391 (2003) [hep-ph/0206071]; H.C. Cheng et al., Phys. Rev. Lett. 89, 211301 (2002) [hep-ph/0207125]; K. Agashe and G. Servant, Phys. Rev. Lett. 93, 231805 (2004) [hep-ph/0403143]; J.A.R. Cembranos et al., Phys. Rev. Lett. 90, 241301 (2003) [hep-ph/0302041].

[18] K.Y. Choi and L. Roszkowski, AIP Conf. Proc. 805, 30 (2006) [hep-ph/0511003]; K.Y. ChоI, L. Roszkowski, R.R de Austri, arXiv:0710. 3349.

[19] J.E. Kiм, Phys. Lett. B 136, 378 (1984);

T. Goto and M. Yamaguchi, ibid. 276, 103 (1992).

[20] R. Peccei And H. Quinn, Phys. Rev. Lett. 38, 1440 (1977);

S. WeInBERG, ibid. 40, 223 (1978);

F. WILCZEK, ibid. 40, 279 (1978).

[21] J. Kıм, Phys. Rep. 150, 1 (1987).

[22] M.Yu. Khlopov and A.D. Linde, Phys. Lett. B 138, 265 (1984); J. Ellis, J.E. Kim and D.V. Nanopoulos, Phys. Lett. B 145, 181 (1984).

[23] T. Moroi, H. Murayama and M. Yamaguchi, Phys. Lett. B 303, 289 (1993); T. MoroI, hep-ph/9503210.

[24] M. Bolz, A. Brandenburg and W. Buchmüller, Nucl. Phys. B606, 518 (2001); (E) ibid. 790, 336 (2008) [hep-ph/0012052].

[25] J. Pradler and F.D. Steffen, Phys. Rev. D 75, 023509 (2007) [hep-ph/0608344].

[26] L. Covi et al., J. High Energy Phys. 05, 033 (2001) [hep-ph/0101009].

[27] A. Brandenburg and F.D. Steffen, J. Cosmol. Astropart. Phys. 08, 008 (2004) [hep-ph/0405158] (version of 17 May 2004).

[28] L. Covi et al., J. High Energy Phys. 07, 023 (2002) [hep-ph/0206119].

[29] V.S. Rychkov and A. Strumia, Phys. Rev. D 75, 075011 (2007) [hep-ph/0701104].

[30] J. Ellis, K.A. Olive, Y. Santoso and V.C. Spanos, Phys. Lett. B 588, 7 (2004) [hep-ph/0312262]; L. Covi et al., J. High Energy Phys. 06, 003 (2004) [hep-ph/0402240].

[31] L. Covi et al., Phys. Rev. Lett. 82, 4180 (1999) [hep-ph/9905212].

[32] T. Asaka And T. Yanagida, Phys. Lett. B 494, 297 (2000) [hep-ph/0006211].

[33] M. Kawasaki, K. Kohri and T. Moroi, Phys. Lett. B 625, 7 (2005) [astro-ph/0402490].

[34] M. Kawasaki, K. Kohri and T. Moroi, Phys. Rev. D 71, 083502 (2005) [astro-ph/0408426]; M. Kawasaki, K. Kohri, T. Moroi, A. Yotsuyanagi, arXiv:0804.3745.

[35] R.H. Cyburt et al., Phys. Rev. D 67, 103521 (2003) [astro-ph/0211258]; J.R. Ellis, K.A. Olive and E. Vangioni, Phys. Lett. B 619, 30 (2005) [astro-ph/0503023].

[36] N. Yoshida et al., Astrophys. J. 591 L1 (2003) [astro-ph/0303622]; K. JedamziK et al., J. Cosmol. Astropart. Phys. 07, 010 (2006) [astro-ph/0508141].

[37] C. Wetterich, Nucl. Phys. B302, 668 (1988).

[38] E.J. Copeland, A.R. Liddle and D. Wands, Phys. Rev. D 57, 4686 (1998) [gr-qc/9711068]; E.J. Copeland, N.J. Nunes and F. Rosati, Phys. Rev. D 62, 123503 (2000) [hep-ph/0005222].

[39] P.J. Steinhardt, L. Wang and I. Zlatev, Phys. Rev. Lett. 82, 896 (1999) [astro-ph/9807002]; ibid., Phys. Rev. D 59, 123504 (1999) [astro-ph/9812313]; S.C.C. NG, N.J. Nunes and F. Rosati, Phys. Rev. D 64, 2001 (083510) [astro-ph/0107321]. 
[40] U. França and R. Rosenfeld, J. High Energy Phys. 10, 015 (2002) [astro-ph/0206194].

[41] J.M. Cline, J. High Energy Phys. 08, 035 (2001) [hep-ph/0105251];

C. Kolda And W. Lahneman, hep-ph/0105300.

[42] J. Weller and A. Albrecht, Phys. Rev. D 65, 103512 (2002) [astro-ph/0106079];

D. Blais and D. Polarski, Phys. Rev. D 70, 084008 (2004) [astro-ph/0404043].

[43] C.L. Gardner, Nucl. Phys. B707, 278 (2005) [astro-ph/0407604]; hep-ph/0701036.

[44] N. OKada and O. Seto, Phys. Rev. D 71, 023517 (2005) [hep-ph/0407235];

E.J. Copeland and O. Seto, Phys. Rev. D 72, 023506 (2005) [hep-ph/0505149].

[45] G. Рanotopoulos, J. Cosmol. Astropart. Phys. 08, 005 (2005) [hep-ph/0506093].

[46] E.W. Kolb and M.S. Turner, The Early Universe, Redwood City, USA: Addison-Wesley (1990).

[47] R.H. Cyburt et al., Astropart. Phys. 23, 313 (2005) [astro-ph/0408033].

[48] R. Bean, S.H. Hansen and A. Melchiorri, Phys. Rev. D 64, 103508 (2001) [astro-ph/0104162]; ibid., Nucl. Phys. 110, (Proc. Suppl.) 167 (2002) [astro-ph/0201127].

[49] G. Bélanger et al., Comput. Phys. Commun. 149, 103 (2002) [hep-ph/0112278]; G. Bélanger, F. Boudjema, A. Pukhov and A. Semenov, hep-ph/0405253.

[50] P. Gondolo et al., J. Cosmol. Astropart. Phys. 07, 008 (2004) [astro-ph/0406204].

[51] M.R. de Garcia Maia, Phys. Rev. D 48, 647 (1993); M.R. de Garcia Maia and J.D. Barrow, Phys. Rev. D 50, 6262 (1994).

[52] M. Giovannini, Phys. Rev. D 60, 123511 (1999) [astro-ph/9903004]; V. Sahni, M. Sami and T. Souradeep, Phys. Rev. D 65, 023518 (2002) [gr-qc/0105121].

[53] T.L. Smith, M. Kamionkowski and A. Cooray, Phys. Rev. D 73, 023504 (2006) [astro-ph/0506422].

[54] J.V. Cunha and J.A.S. Lima, arXiv:0805.1261.

[55] E.W. Kolb and S. Wolfram, Nucl. Phys. B172, 224 (1980).

[56] Particle Data Group, Phys. Lett. B 592, 104 (2004).

[57] J. Edsü and P. Gondolo, Phys. Rev. D 56, 1879 (1997) [hep-ph/9704361].

[58] G. Lazarides, R.K. Schaefer, and Q. Shafi, Phys. Rev. D 56, 1324 (1997) [hep-ph/9608256].

[59] S. Chang And H.B. Kim, Phys. Rev. Lett. 77, 591 (1996) [hep-ph/9604222]; M. Kawasaki et al., J. Cosmol. Astropart. Phys. 03, 009 (2008) [arXiv: 0711. 3083].

[60] K. Rajagopal, M.S. Turner and F. Wilczek, Nucl. Phys. B358, 447 (1991).

[61] R.J. Scherrer and M.S. Turner, Phys. Rev. D 31, 681 (1985); C. Pallis, Astropart. Phys. 21, 689 (2004) [hep-ph/0402033]; C. Pallis, Nucl. Phys. B751, 129 (2006) [hep-ph/0510234].

[62] R. Rangarajan and N. Sahu, hep-ph/0606228.

[63] M. Sami and N. Dadhich, TSPU Vestnik 44N7, 25 (2004) [hep-th/0405016].

[64] E.J. Chun and S. Scopel, J. Cosmol. Astropart. Phys. 10, 011 (2007) [arXiv: 0707. 1544].

[65] M.C. Bento et al., Phys. Rev. D 73, 023506 (2006) [hep-ph/0508213]; N. OKada and O. Seto, Phys. Rev. D 73, 063505 (2006) [hep-ph/0507279].

[66] X.J. Bi, M.Z. Li and X.M. Zhang, Phys. Rev. D 69, 123521 (2004) [hep-ph/0308218]; X.J. Bi, J.X. Wang, C. Zhang and X. Zhang, Phys. Rev. D 70, 123512 (2004) [hep-ph/0404263].

[67] J. Ellis et al., Astropart. Phys. 13, 181 (2000) (E) ibid. 15, 413 (2001) [hep-ph/9905481].

[68] H. Haber and G. L. Kane, Phys. Rep. 117, 75 (1985).

[69] J. PradLER, arXiv:0708.2786.

[70] R. Cutler and D. Sivers, Phys. Rev. D 17, 1978 (196).

[71] W. Beenakker, R. Hopker and P.M. Zerwas, Phys. Lett. B 378, 159 (1996) [hep-ph/9602378].

[72] A. Puкноv, hep-ph/0412191. 